\title{
IMPROVEMENT OF THE RICHNESS ESTIMATES OF maxBCG CLUSTERS
}

\author{
Eduardo Rozo ${ }^{1}$, Eli S. RykofF ${ }^{2,13}$, Benjamin P. Koester ${ }^{3,4}$, Timothy McKay ${ }^{5,6,7}$, Jiangang HaO ${ }^{5}$, August Evrard ${ }^{5,6,7}$, \\ Risa H. Wechsler ${ }^{8}$, SARah HANSEN ${ }^{3,4}$, Erin Sheldon ${ }^{9}$, David Johnston ${ }^{10}$, Matthew BeCKer ${ }^{3,4}$, James AnNis ${ }^{11}$, \\ LiNDSEY BLEEM $^{3}$, AND RyAN SCRANTON ${ }^{12}$ \\ ${ }^{1}$ Center for Cosmology and Astro-Particle Physics (CCAPP), The Ohio State University, Columbus, OH 43210, USA \\ ${ }^{2}$ Physics Department, University of California at Santa Barbara, 2233B Broida Hall, Santa Barbara, CA 93106, USA \\ ${ }^{3}$ Department of Astronomy and Astrophysics, The University of Chicago, Chicago, IL 60637, USA \\ ${ }^{4}$ Kavli Institute for Cosmological Physics, The University of Chicago, Chicago, IL 60637, USA \\ ${ }^{5}$ Physics Department, University of Michigan, Ann Arbor, MI 48109, USA \\ ${ }^{6}$ Astronomy Department, University of Michigan, Ann Arbor, MI 48109, USA \\ ${ }^{7}$ Michigan Center for Theoretical Physics, Ann Arbor, MI 48109, USA \\ ${ }^{8}$ Kavli Institute for Particle Astrophysics \& Cosmology, Physics Department, and Stanford Linear Accelerator Center, Stanford University, Stanford, CA 94305, USA \\ ${ }^{9}$ Center for Cosmology and Particle Physics, Physics Department, New York University, New York, NY 10003, USA \\ 10 Jet Propulsion Laboratory, 4800 Oak Grove Drive, Pasadena, CA 91109, USA \\ ${ }^{11}$ Fermi National Accelerator Laboratory, P.O. Box 500, Batavia, IL 60510, USA \\ 12 Department of Physics and Astronomy, University of Pittsburgh, 3941 O'Hara St., Pittsburgh, PA 15260, USA \\ Received 2008 September 16; accepted 2009 May 26; published 2009 August 31
}

\begin{abstract}
Minimizing the scatter between cluster mass and accessible observables is an important goal for cluster cosmology. In this work, we introduce a new matched filter richness estimator, and test its performance using the maxBCG cluster catalog. Our new estimator significantly reduces the variance in the $L_{X}-$ richness relation, from $\sigma_{\ln L_{X}}^{2}=(0.86 \pm 0.02)^{2}$ to $\sigma_{\ln L_{X}}^{2}=(0.69 \pm 0.02)^{2}$. Relative to the maxBCG richness estimate, it also removes the strong redshift dependence of the $L_{X}$-richness scaling relations, and is significantly more robust to photometric and redshift errors. These improvements are largely due to the better treatment of galaxy color data. We also demonstrate the scatter in the $L_{X}$-richness relation depends on the aperture used to estimate cluster richness, and introduce a novel approach for optimizing said aperture which can easily be generalized to other mass tracers.
\end{abstract}

Key words: cosmology: observations - methods: data analysis

Online-only material: color figures

\section{INTRODUCTION}

The dependence of the halo mass function on cosmology is a problem that is well understood both analytically (Press \& Schechter 1974; Bond et al. 1991; Sheth \& Tormen 2002) and numerically (Jenkins et al. 2001; Warren et al. 2006; Tinker et al. 2008). In principle, this detailed understanding allows one to place tight constraints on the amplitude of the primordial power spectrum and on dark energy parameters (e.g., Holder et al. 2001; Haiman et al. 2001). In practice, life is not so simple. Cluster mass is not an observable, ${ }^{14}$ and so we must rely on other quantities that trace mass to estimate the halo mass function. In this context, observables that are tightly correlated with mass and whose scatter is well understood are highly desirable, as they permit a more accurate measurement of the mass function.

One such mass tracer, and the subject of interest for this work, is the so-called cluster richness, a measure of the galaxy content of a cluster. ${ }^{15}$ Relative to other popular mass tracers such as

\footnotetext{
13 TABASGO Fellow

14 Here, we take the view that a cluster "observable" is a quantity that is readily available with almost no systematic uncertainties in its interpretation. In our mind, cluster mass is not an observable because all mass estimators are subject to important systematics, in a way that more direct observables such as galaxy velocity dispersions or X-ray luminosities are not.

15 Throughout this work, the word "richness" is meant to be taken as shorthand for "optical mass tracer," and not as the actual number of cluster galaxies within the virialized region of a cluster. Examples of cluster richness include those discussed in this work, the amplitude of the cluster-mass correlation function $B \mathrm{gc}$, the optical luminosity of a cluster (which can itself be defined in many ways), etc.
}

X-ray properties, SZ-decrements, and galaxy velocity dispersion, optical richness has unique advantages and disadvantages. Its unique advantages are the following.

1. Cluster richness can easily be estimated with inexpensive, photometric optical data.

2. Cluster richness can be estimated for both massive clusters and low-mass groups.

The first of these two properties is significant because it implies that cluster richness estimates are readily available given any large, photometric optical survey such as the Sloan Digital Sky Survey (SDSS; York et al. 2000a), DES, ${ }^{16}$ Pan-STARRS, ${ }^{17}$ or LSST. ${ }^{18}$ The latter property, on the other hand, is an important advantage for a much more interesting reason.

It has long been known that cosmological constraints from galaxy clusters are degenerate in $\sigma_{8}$ and $\Omega_{m}$, a condition that is usually summarized as $\sigma_{8} \Omega_{m}^{\gamma}=$ constant (see, e.g., Mantz et al. 2008; Henry et al. 2009; Vikhlinin et al. 2009b; Rozo et al. 2009, for the most recent analysis). Here, $\gamma \approx 0.5, \sigma_{8}$ is a parameter specifying the amplitude of the primordial power spectrum, and $\Omega_{m}$ is the matter density of the universe in units of the critical density. The existence of this degeneracy is easy to explain (Rozo et al. 2004): suppose that we only measured the abundance of galaxy clusters at a single mass scale. Since the halo mass function depends on both $\sigma_{8}$ and $\Omega_{m}$, it is evident that with just one observable there must be a degeneracy between

\footnotetext{
16 http://www.darkenergysurvey.org/

$17 \mathrm{http}: / /$ pan-starrs.ifa.hawaii.edu/public/

18 http://www.lsst.org/lsst_home.shtml
} 
these two parameters. But what if we measure the halo mass function over a range of scales? This is roughly equivalent to measuring the amplitude and slope of the halo mass function at the statistical pivot point. If the mass range probed is small, then the slope of the mass function is not well constrained, and the degeneracy between $\sigma_{8}$ and $\Omega_{m}$ will remain. In order to break this degeneracy using only cluster abundance data requires a measurement of the halo mass function over a large range of masses or a large redshift range. Given local cluster samples, only spectroscopic velocity measurements and optical richness estimates can probe a mass range wide enough to successfully break this degeneracy, but the former requires considerably more observing resources.

There are, however, important disadvantages in using cluster richness as a mass tracer. For instance, historically, the fact that the relation between cluster richness and mass cannot be predicted a priori based on simple physical arguments was viewed as a significant drawback. Nowadays, however, this argument holds little sway, since the level of accuracy required for precision cosmology in our a priori knowledge of cluster scaling relations is pushing current research toward a selfcalibrating approach, in which both cosmology and cluster scaling relations are simultaneously constrained from the data (Lima \& Hu 2004; Majumdar \& Mohr 2004; Lima \& Hu 2005; Hu \& Cohn 2006; Wu et al. 2008). Thus, the lack of a simple physical model for predicting cluster richness is no longer a serious drawback as self-calibration techniques are viewed as desirable, even in the case of a priori knowledge of cluster scaling relations.

Another reason why optical richness estimates fell out of favor relative to other mass tracers is that, in the past, richness estimates were known to suffer from significant projection effects, which resulted in impure cluster samples as well as large scatter in the mass-richness relation. Abell made one of the first systematic attempts at measuring richness (Abell 1958; Abell et al. 1989) in defining his richness classes. He tried to minimize projection by only counting galaxies dimmer than $m_{3}$, the magnitude of the third brightest cluster galaxy, but brighter than $m_{3}+2$. The bright cut is aimed at foreground interlopers, while the dim cut reduces the contribution of the galaxy background. Since then, new algorithms have been developed, and many of them have been applied to CCD-based imaging (e.g., matchedfilter methods Postman et al. 1996; Bramel et al. 2000; Yee \& López-Cruz 1999; Kochanek et al. 2003; Dong et al. 2008).

Projection effects are now a much more benign problem thanks to these new richness measurement techniques, the advent of accurate photometric data enabled by modern CCDs, and most recently, the exploitation of the well known observation that ellipticals and cluster $\mathrm{E} / \mathrm{S} 0$ galaxies in particular tend to form a tight ridgeline in color-magnitude space (Visvanathan \& Sandage 1977; Bower et al. 1992; Gladders \& Yee 2000; Koester et al. 2007b). This color clustering has been indispensable to richness measurements in the SDSS (Goto et al. 2002; Miller et al. 2005; Koester et al. 2007b) and the Red Sequence Cluster Survey (RCS: Gladders \& Yee 2005), and such colorbased measures have been shown to be effective mass tracers (Muzzin et al. 2007; Sheldon et al. 2007a; Johnston et al. 2007; Rykoff et al. 2008b; Becker et al. 2007a).

While richness estimates show a strong correlation with other mass proxies (e.g., Bahcall 1981; Yee \& Ellingson 2003; Gilbank et al. 2004; Dai et al. 2007; Lopes et al. 2006; Sheldon et al. 2007a; Johnston et al. 2007; Becker et al. 2007a; Rykoff et al. 2008a), considerable scatter in the mass-richness relation still remains. For instance, the richness measure used in the RCS cluster catalog has a logarithmic scatter of $\sigma_{\ln M} \approx 0.8$ (Gladders et al. 2007), while for maxBCG clusters the number is closer to $\sigma_{\ln M} \approx 0.5$ (Rozo et al. 2008). This is to be compared with the scatter for X-ray mass tracers, which is expected to be as low as $\sigma_{\ln M}=0.08$ for $Y_{X}$ based on simulations (Kravtsov et al. 2006), or as high as $\sigma_{\ln M}=0.25$ for non-core extracted soft X-ray band luminosities (e.g., Stanek et al. 2006; Vikhlinin et al. 2009a). Clearly, much improvement is needed to bring the scatter of richness measures to the level of X-ray mass tracers. ${ }^{19}$

This work is aimed at reducing the variance in the richnessmass relation. We do this by explicitly constructing a new richness estimator that significantly reduces the scatter in mass at fixed richness for maxBCG clusters. Relative to $N_{200}$ of maxBCG, we introduce two significant differences. The first of these involves using a matched filter algorithm to estimate cluster richness. Matched filters have been used in the literature before (Postman et al. 1996; Kochanek et al. 2003). Unlike those works, however, our matched filter includes a color component, which is of critical importance for reducing projection effects over the redshift range spanned by our cluster sample. Indeed, we find that most of the improvement we observe in our richness estimator is driven by our improved modeling of galaxy colors when estimating cluster richness. In some sense, our filter is close in spirit to that of Dong et al. (2008), who include a photometric redshift filter into their richness estimate. We also note here that group-scale studies suggest that some measure of the average color in the cluster is indicative of mass, particularly below $\sim 10^{14} M_{\odot}$ (Martínez et al. 2002; Martínez \& Muriel 2006; Weinmann et al. 2006; Hansen et al. 2007).

The second difference we introduce is the way in which the aperture used to estimate cluster richness is determined. Generically, cluster richness estimators involve counting the number of galaxies within some specified aperture, which can thus be interpreted as defining the "size" of the cluster. This begs the question, then, of how is one to select the correct size of a cluster a priori? Theoretically, halo sizes are usually defined in terms of $R_{\Delta}$, a radius which encompasses a mean density that is $\Delta$ times either the mean or the critical density of the universe (conventions vary from author to author). Unfortunately, not only is such a definition not applicable observationally, ${ }^{20}$ authors vary both on the reference background density (critical versus mean mass density), and on the specific overdensity value. Thus, even though significant progress has been made (Cuesta et al. 2008), a definitive definition of halo size remains elusive.

In this work, we approach this question with observations in mind. That is, rather than coming with a preconceived notion of what the radius of a cluster is, we let the data tell us what the optimal radii for our clusters is by demanding that optical richness be as tightly correlated as possible with $\mathrm{X}$-ray luminosity. This approach was pioneered by Popesso et al. (2004) and Lopes et al. (2006), who use the scatter in the $L_{X}$-richness relation to derive an ideal fixed aperture with which to estimate optical richness. Here, however, we wish to allow for the fact that larger clusters are expected to have larger radii, which suggests that a fixed aperture is not ideal. To explore this possibility, we proceed as follows: first, one posits

\footnotetext{
19 In all scatter values quoted above, scatter is defined as the standard deviation in $\log$ mass at a fixed observable.

${ }^{20}$ By this we mean that to compute $M_{\Delta}$ one needs to know the background density or the critical density at the redshift of the cluster. Either requires us to know the underlying cosmology a priori, which is impossible. Thus, the quoted values for $M_{\Delta}$ always have an explicit cosmology assumption in them.
} 
a scaling relation between cluster richness and cluster radius. When estimating cluster richness, one then demands that the richness-radius scaling relation be satisfied. For instance, given a cluster, one can simply make an initial guess for its richness. Using the richness-radius scaling relation, one can then draw a circle of the appropriate radius, and count the number of galaxies within it. If the richness was underestimated, one will find too few galaxies, signaling that the richness estimate must be increased. Proceeding in this way, one can quickly zero in on the appropriate richness for the object.

This does, however, leave open the question of what the correct richness-radius relation is. Since we are interested in finding a new richness estimator that is tightly correlated with halo mass, we can use the scatter in the mass-richness relation as our figure of merit to determine the "correct" richness-radius relation. In practice, we use the $L_{X}$-richness scatter rather than the mass-richness scatter because we cannot measure the scatter in mass directly. We emphasize that since the mass scatter at fixed X-ray luminosity (see, e.g., Vikhlinin et al. 2009a) is considerably tighter than the corresponding scatter at fixed richness (Rozo et al. 2008), the use of X-ray luminosity as a mass tracer for our purposes is well justified.

The layout of the paper is as follows. We describe the data sets used in this work in Section 2. Our matched filter estimator is introduced in Section 3, followed by our method for determining the optimal radius-richness relation in Section 4. We present our results in Section 5. In investigating the properties of our new richness measure, we have discovered that the redshift evolution of the $L_{X}$-richness relation of our new estimator is much milder than that measured for $N_{200}$. These results and the corresponding discussion are presented in Section 6. We summarize our results and present our conclusions in Section 7. Throughout, whenever needed a flat $\Lambda$ CDM cosmology with $\Omega_{M}=0.3$ and $h=1.0$ was assumed.

\section{DATA}

The data for the analysis presented in this work come from two large area surveys, the SDSS (York et al. 2000b) and the ROSAT All-Sky Survey (RASS; Voges et al. 1999). SDSS imaging data are used to select clusters and to measure their matched filter richness; RASS data provide $0.1-2.4 \mathrm{keV}$ X-ray fluxes, which we convert into estimates of the $\mathrm{X}$-ray luminosity of the clusters.

\subsection{SDSS}

The imaging and spectroscopic surveys that comprise the SDSS are currently in the sixth Data Release (AdelmanMcCarthy et al. 2008). This release includes nearly $8500 \mathrm{deg}^{2}$ of drift-scan imaging in the Northern Galactic Cap, and another $7500 \mathrm{deg}^{2}$ of spectroscopic observations of stars, galaxies, and quasars.

The camera design (Gunn et al. 2006) and drift-scan imaging strategy of the SDSS enable acquisition of nearly simultaneous observations in the $u, g, r, i, z$ filter system (Fukugita et al. 1996). Calibration (Hogg et al. 2001; Smith et al. 2002; Tucker et al. 2006), astrometric (Pier et al. 2003), and photometric (Lupton et al. 2001) pipelines reduce the data into object catalogs containing a host of measured parameters for each object.

The maxBCG cluster sample and the galaxy catalogs used to remeasure cluster richness in this paper are derived from the SDSS. The galaxy catalogs are drawn from an area approximately coincident with DR4 (Adelman-McCarthy et al. 2006). Galaxies are selected from SDSS object catalogs as described in
Sheldon et al. (2007b). In this work we use CMODEL_COUNTS as our total magnitudes, and MODEL_COUNTS when computing colors. The latter choice is made to ensure that the magnitudes used to compute colors are estimated with the same angular aperture in the different bands, so that the color we obtain is a measure of the mean color of the galaxy at a specific radius. Bright stars, survey edges and regions of poor seeing are masked as previously described (Koester et al. 2007a; Sheldon et al. 2007b).

\subsection{Cluster Sample}

We obtain sky locations, redshift estimates, and initial richness values from the maxBCG cluster catalog. Details of the selection algorithm and catalog properties are published elsewhere (Koester et al. 2007a, 2007b). In brief, maxBCG selection relies on the observation that the galaxy population of clusters is dominated by luminous, red galaxies clustered tightly in color (the E/S0 ridgeline). Since these galaxies have old, passively evolving stellar populations, their $g-r$ color closely reflects their redshift. The brightest such red galaxy, typically located at the peak of the galaxy density, defines the cluster center.

The maxBCG catalog is approximately volume limited in the redshift range $0.1 \leqslant z \leqslant 0.3$, with very accurate photometric redshifts $(\delta z \sim 0.01)$. Studies of the maxBCG algorithm applied to mock SDSS catalogs indicate that the completeness and purity are very high, above $90 \%$ (Koester et al. 2007a; Rozo et al. 2007). The maxBCG catalog has been used to investigate the scaling of galaxy velocity dispersion with cluster richness (Becker et al. 2007b) and to derive constraints on the power spectrum normalization, $\sigma_{8}$, from cluster number counts (Rozo et al. 2007).

The primary richness estimator used in the maxBCG catalog is $N_{200}$, defined as the number of galaxies with $g-r$ colors within $2 \sigma$ of the E/S0 ridgeline as defined by the BCG color, brighter than $0.4 L_{*}$ (in the $i$ band), and found within $r_{200}^{\mathrm{gal}}$ of the cluster center. $r_{200}^{\mathrm{gal}}$ is a cluster radius that depends upon the richness $N_{\text {gals }}$, which is defined exactly as $N_{200}$ with the exception of the radius employed, which is now taken to be a fixed metric aperture of $1 h^{-1} \mathrm{Mpc}$. The radius $r_{200}$ is calibrated so that, on average, the galaxy overdensity within $r_{200}^{\text {gal }}$ is $200 \Omega_{m}^{-1}$ assuming $\Omega_{m}=0.3$ (Hansen et al. 2005). The full catalog comprises 13,823 objects with a richness threshold $N_{200} \geqslant 10$, corresponding to $M \gtrsim 5 \times 10^{13} h^{-1} M_{\odot}$ (Johnston et al. 2007).

As mentioned in the introduction, we re-estimate the cluster richness for every object in the maxBCG catalog, and measure the corresponding scatter in the $L_{X}$-richness relation. When doing so, we always limit ourself to the 2000 richest clusters, ranked according to the new richness estimate. This cut is made to ensure that our results are insensitive to the $N_{200} \geqslant 10$ cut of the maxBCG catalog. This is a somewhat subtle point: to estimate the scatter in the $L_{X}-\lambda$ relation, we wish to use a $\lambda$ thresholded sample with $\lambda>\lambda_{\text {min }}$. In order to define such a sample from the maxBCG, one must require that there be no clusters with $\lambda>\lambda_{\min }$ and $N_{200} \leqslant 10$, the maxBCG richness cut. This condition is satisfied as long as we limit ourselves to about the richest 2000 clusters. We have explicitly checked that our results are insensitive to selecting fewer than 2000 clusters, but our results become noisier. We have also explicitly checked that we cannot use much more than 2000 clusters before our results start becoming affected by our choice of $\lambda_{\min }$. We also note that our choice of always selecting the 2000 richest clusters also implies that the specific cluster sample used to estimate the scatter in the $L_{X}$-richness relation 
varies somewhat as we vary the parameters of the richness estimator.

\subsection{X-ray Measurements}

The scatter in $L_{X}$ at fixed richness is estimated using a slight variant of the method presented in Rykoff et al. (2008b). Briefly, we use the RASS photon maps to estimate the $0.5-2.0 \mathrm{keV}$ $\mathrm{X}$-ray flux at the location of each cluster, which is used to derive $L_{X}$ [0.1-2.4 keV] using the cluster photometric redshift (the conversion factors are similar to those used in Böhringer et al. 2004). We then perform a Bayesian linear least-squares fit to $\ln L_{x}$ as a function of $\ln N$, where $N$ is the richness parameter to be tested. The variance in $\ln L_{X}$ is included as a free parameter. The fit is done following the algorithm presented in Kelly (2007), and correctly takes into account upper limits for $L_{X}$ for those clusters with upper limits on X-ray emission.

It is important to note here that the estimated X-ray luminosity of a cluster depends on the aperture used to measure $L_{X}$. Rykoff et al. (2008b) used a fixed $750 \mathrm{~h}^{-1} \mathrm{kpc}$ aperture as a compromise between needing a large aperture to avoid losing Xray photons due to the ROSAT point spread function (PSF) and cluster miscentering, and the need for a small aperture in order to increase the signal-to-noise of the cluster emission. Since, we have discovered that the scatter in the $L_{X}-N_{200}$ relation is minimized when we measure $L_{X}$ within a $1 h^{-1}$ Mpc radius. ${ }^{21}$ The corresponding scatter for the top 2000 maxBCG clusters is $\sigma_{\ln L_{X} \mid N_{200}}=0.96 \pm 0.03$. $^{22}$

The nature of the present exercise has the benefit of assigning a cluster radius $R_{c}$, to each individual cluster, so it is natural to measure $L_{X}$ in the same scale as the optical richness. Thus, in this work, we estimate $L_{X}$ using a variable aperture which depends upon the cluster's richness. Using a fixed $1 h^{-1} \mathrm{Mpc}$ aperture to estimate $L_{X}$ does not have a large effect on our results, for reasons that will be discussed below. Finally, we note that very small physical apertures are impractical for the most distant clusters due to the large size of the RASS PSF, which corresponds to a physical scale of $300 h^{-1} \mathrm{kpc}(\mathrm{FWHM})$ at $z=0.23$, the median redshift of the maxBCG catalog. Therefore, we place a fixed minimum aperture of $500 h^{-1} \mathrm{kpc}$ for each cluster. We discuss the small effect of this aperture cutoff in Section 4.

\subsection{Cleaning the Sample}

Our analysis depends on a combination of optical and X-ray measurements of maxBCG clusters using SDSS and RASS data. As discussed in detail in Rykoff et al. (2008b, see Section 5.6), there is clear evidence that cool core clusters increase the scatter in X-ray cluster properties. High-resolution X-ray imaging of clusters allows the exclusion of cluster cores, reducing the scatter in observed X-ray properties (e.g., O'Hara et al. 2006; Chen et al. 2007; Maughan 2007). Unfortunately, the broad PSF of RASS means that it is impossible to exclude the cores of clusters in this work. In order to asses how robust our results are to the presence or absence of cooling flow clusters in the

\footnotetext{
21 This is in essence the converse procedure to that explored in Popesso et al. (2004) and Lopes et al. (2006), in that we hold the richness definition fixed, and we then vary the aperture used to estimate $L_{X}$.

22 The attentive reader will note that the quoted scatter in $L_{X}$ at fixed richness is significantly larger than the scatter in mass at fixed richness quoted in the introduction, which was closer to 0.5 . Given a slope of $\approx 1.6$ in the $L_{X}-M$ relation, a scatter of 0.96 in $L_{X}$ corresponds to $\approx 0.96 / 1.6 \approx 0.6$ scatter in mass. The remaining $10 \%$ difference is because the scatter Rozo et al. (2008) uses the scatter of the 1000 richest clusters, which is smaller than that of the 2000 richest clusters by 0.1 .
}

cluster sample, we have created a "clean" sample of maxBCG clusters by removing all known cool core clusters that might have boosted global X-ray luminosity and may significantly bias our results. In addition, we have removed apparently X-ray bright maxBCG clusters that were determined via inspection to have their X-ray flux significantly contaminated by foreground objects such as stars, low redshift galaxy clusters, and active galactic nuclei (AGNs).

There does not exist a complete, unbiased catalog of cool core X-ray clusters. Peres et al. (1998) used ROSAT pointed observations of an X-ray-flux-limited catalog (Edge et al. 1990) to estimate the central cooling time of 55 nearby clusters. Two of these are in the maxBCG catalog (A1689, A2244) and exhibit characteristics of a cool core $\left(t_{\text {cool }}<10 \mathrm{Gyr}\right)$. Bauer et al. (2005) used Chandra observations of the higher redshift $(z>0.15)$ clusters to systematically estimate the central cooling time of bright X-ray clusters. We identify seven maxBCG clusters (A750, A963, A1835, RXJ 2129.6+0005, Z2701, Z3146, Z7160) from this sample with cooling times $t_{\text {cool }}<10 \mathrm{Gyr}$ which we mark as cool core clusters. Finally, Böhringer et al. (2005) identified ClG J1504.1-0248 as a cluster with a massive cooling core. These clusters are typically very $\mathrm{X}$-ray bright for their richness, and tend to show strong $H \alpha$ emission in the SDSS spectroscopy. In fact, for several of these clusters maxBCG failed to identify the correct BCG because the strong $H \alpha$ emission changes the apparent galaxy color, making it inconsistent with the red sequence of the cluster. From here on, the maxBCG catalog presented in Koester et al. (2007b) is referred to as the "full" cluster sample, and the subsample with these cooling core cluster removed is referred to as the "clean" cluster sample.

\section{MATCHED FILTER RICHNESS ESTIMATORS}

\subsection{Derivation of the Matched Filter Richness Estimator}

Let $\mathbf{x}$ be a vector characterizing the observable properties of a galaxy (e.g., galaxy color and magnitude). We model the projected galaxy distribution around clusters as a sum $S(\mathbf{x})=\lambda u(\mathbf{x} \mid \lambda)+b(\mathbf{x})$ where $\lambda$ is the number of cluster galaxies, $u(\mathbf{x} \mid \lambda)$ is the cluster's galaxy density profile normalized to unity, and $b(\mathbf{x})$ is the density of the background (i.e., non-member) galaxies. The probability that a galaxy found near a cluster is actually a cluster member is given by

$$
p(\mathbf{x})=\frac{\lambda u(\mathbf{x} \mid \lambda)}{\lambda u(\mathbf{x} \mid \lambda)+b(\mathbf{x})} .
$$

Consequently, the total number of cluster galaxies $\lambda$ must satisfy the constraint equation

$$
\lambda=\sum p(\mathbf{x} \mid \lambda)=\sum \frac{\lambda u(\mathbf{x} \mid \lambda)}{\lambda u(\mathbf{x} \mid \lambda)+b(\mathbf{x})},
$$

where the sum is over all galaxies in the cluster field. If the filters $u(\mathbf{x} \mid \lambda)$ and $b(\mathbf{x})$ are known, then given an observed galaxy distribution $\left\{\mathbf{x}_{1}, \ldots, \mathbf{x}_{N}\right\}$ around a cluster we can define a richness estimator $\hat{\lambda}$ as the solution to Equation (2). As it turns out, one can also derive this expression using a maximum likelihood (ML) approach, and indeed, this is how the estimator first arose (Postman et al. 1996). ${ }^{23}$ Interested readers are referred

\footnotetext{
23 The estimator derived here is not actually the same as the Postman et al (1996) estimator. The main difference is that ours represents the "fine grained likelihood" estimator, while Postman et al. (1996) employed the "coarsed grain likelihood" approximation in their derivation (see Kepner et al. 1999, for further discussion).
} 
to Appendix A for details. From now on, the letter $\lambda$ shall always refer to a matched filter richness estimate obtained with Equation (2).

\subsection{Cluster Radii and Matched Filter Richness Estimates}

Consider again Equation (2). As mentioned before, the sum used in Equation (2) needs to extend over all galaxies. In practice, of course, one needs to add over all galaxies within some cutoff radius $R_{c}$. Operationally, this is equivalent to setting $u=0$ for all galaxies with radii $R>R_{c}$, so it is natural to interpret the cutoff radius $R_{c}$ as a cluster radius. In this light, it seems obvious that considerable care must be taken to choose the correct cluster radius when estimating richness, but how to go about doing just that is a less straightforward question (Popesso et al. 2004; Lopes et al. 2006).

In this work, we propose that cluster radii be selected on the basis of a model radius-richness relation. Specifically, we assume that the size of a cluster of richness $\lambda$ scales as a power law of $\lambda$,

$$
R_{c}(\lambda)=R_{0}(\lambda / 100.0)^{\beta} .
$$

Naively, we expect $R_{0} \approx 1 \mathrm{Mpc}$, as that is the characteristic size of clusters, and $\beta \approx 1 / 3$ assuming that $R \propto M^{1 / 3} \propto \lambda^{1 / 3}$. We postpone the discussion of how we go about selecting $R_{0}$ and $\beta$ in Section 4 . For the time being, we shall simply assume that $R_{0}$ and $\beta$ are known. In that case, Equation (2) becomes

$$
\lambda=\sum p(\mathbf{x} \mid \lambda)=\sum_{R<R_{c}(\lambda)} \frac{\lambda u(\mathbf{x} \mid \lambda)}{\lambda u(\mathbf{x} \mid \lambda)+b(\mathbf{x})} .
$$

Note that we have explicitly included the cutoff radius $R_{c}$ in the sum above, and that this cutoff radius now depends on $\lambda$. We emphasize that in the above equation, the cluster richness $\lambda$ is the only unknown, so we can numerically solve for $\lambda$. That is, our richness estimator $\lambda$ is defined as the solution to $f(\lambda)=0$ where $^{24}$

$$
0=f\left(\lambda \mid R_{0}, \beta\right)=\lambda-\sum_{R<R_{c}(\lambda)} \frac{\lambda u(\mathbf{x} \mid \lambda)}{\lambda u(\mathbf{x} \mid \lambda)+b(\mathbf{x})} .
$$

Note that because $R_{c}(\lambda)$ depends on both $R_{0}$ and $\beta$, the solution to $f(\lambda)=0$ will itself depend on $R_{0}$ and $\beta$. That is, any two values for these two parameters define a unique and distinct richness estimator.

Figure 1 shows the function $f\left(\lambda \mid R_{0}, \beta\right)$ for cluster SDSS J082026.8+073650.1 for two different values of $R_{0}$ and $\beta$ : these are $R_{0}=1.27 h^{-1} \mathrm{Mpc}$ and $\beta=1 / 3$, and $R_{0}=1 h^{-1} \mathrm{Mpc}$ and $\beta=0.0$. We note that in both cases there is a unique zero crossing of the function $f(\lambda)$, implying that our richness estimator is well defined, but that the value of this zero crossing depends on $R_{0}$ and $\beta$. We discuss below how we go about selecting optimal $R_{0}$ and $\beta$ values.

Before we move on, it is perhaps worth illustrating the above formalism with a simple toy model. Consider a simple model in which the probability $p$ of a galaxy being a cluster member is a simple top-hat: membership probability is simply

$$
p(R \mid \lambda)=\left\{\begin{array}{lc}
1 & \text { if } R \leqslant R_{c}(\lambda) \\
0 & \text { otherwise. }
\end{array}\right.
$$

\footnotetext{
24 Note that since we are explicitly setting $u=0$ for $R>R_{c}$, the fact that $u$ must be normalized to unity necessarily introduces a dependence of $u$ on $\lambda$ That is, changing $\lambda$ will not only change the range of the sum in Equation (4), but will also change the value of the summands.
}

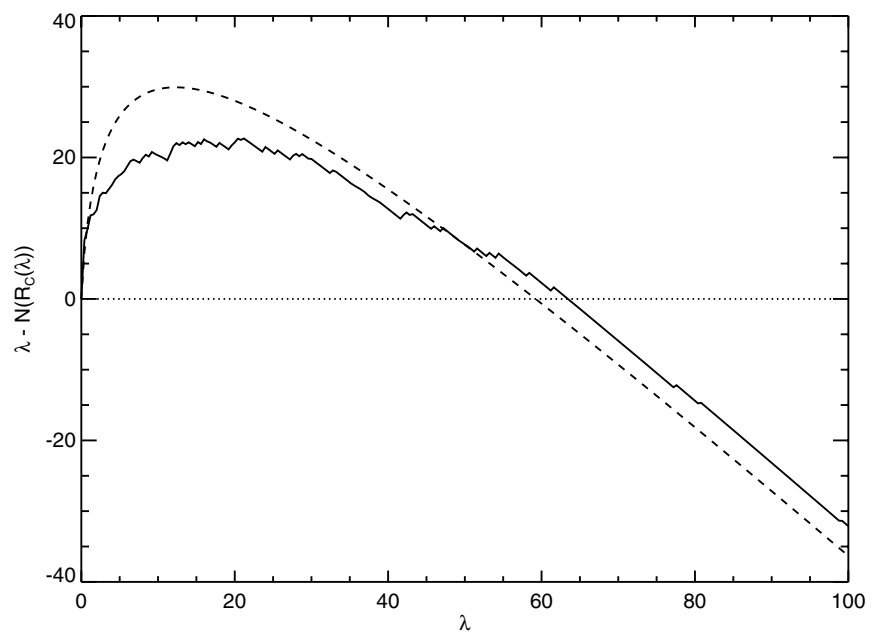

Figure 1. Function $f\left(\lambda \mid R_{0}, \beta\right)$ defined in Equation (5) for two different values of $R_{0}$ and $\beta$ for the cluster SDSS J082026.8+073650.1. The values for $R_{0}$ and $\beta$ are $R_{0}=1.27 h^{-1} \mathrm{Mpc}$ and $\beta=1 / 3$ (solid), and $R_{0}=0.9 h^{-1} \mathrm{Mpc}$ and $\beta=0$ (dashed). Our richness estimator is defined as the solution to the equation $f\left(\lambda \mid R_{0}, \beta\right)=0$. Every pair of values $\left(R_{0}, \beta\right)$ defines a unique estimator. The fact that the two curves above result in similar richness estimates is by design. Section 4 describes how we go about optimizing the values for $R_{0}$ and $\beta$.

We further assume that $R_{0}=1 h^{-1} \mathrm{Mpc}$ and $\beta=0$. The function $f(\lambda)$ is therefore

$$
f(\lambda)=\lambda-N\left(1 h^{-1} \mathrm{Mpc}\right)
$$

where $N(R)$ is the observed number of galaxies within $R$ of the cluster center. Our richness estimator is given by $f(\lambda)=0$, or, solving for $\lambda, \lambda=N\left(1 h^{-1} \mathrm{Mpc}\right)$. This is as expected: if our cluster model is that all galaxies within a radius $R=1 h^{-1} \mathrm{Mpc}$ of the cluster center are cluster galaxies, then the richness estimate of a cluster is simply the total number of galaxies within the said radius. The only difference between this simple example and our proposed richness estimator is the way we estimate membership probabilities, and the values of $R_{0}$ and $\beta$ used to estimate cluster richness.

\subsection{The Filters}

In this work we consider three observable properties of galaxies: $R$, the projected distance from a galaxy to the assigned cluster center, $m$, the galaxy magnitude, and $c$, the galaxies' $g-r$ color. We adopt a separable filter function

$$
u(\mathbf{x})=[2 \pi R \Sigma(R)] \phi(m) G(c),
$$

where $\Sigma(R)$ is the two-dimensional cluster galaxy density profile, $\phi(m)$ is the cluster luminosity function (expressed in apparent magnitudes), and $G(c)$ is the color distribution of cluster galaxies. The prefactor $2 \pi R$ in front of $\Sigma(R)$ accounts for the fact that given $\Sigma(R)$, the radial probability density distribution is given by $2 \pi R \Sigma(R)$. Also, note that the separability condition makes the implicit assumption that these three quantities are fully independent of each other, which is not true in detail (for a discussion of the galaxy population of maxBCG clusters see Hansen et al. 2007). For instance, the tilt of the ridgeline implies that the mean color of a red-sequence cluster galaxy varies slightly as a function of magnitude. We postpone an investigation of how including the correlation between these various observables affects our conclusions to future work (B. P. Koester et al. 2009, in preparation). We now describe each of our three 
filters in detail. We note that defining said filters requires us to specify parameters governing the shape of the filters (e.g., $R_{s}$ for the radial filter, $\alpha$ for the luminosity filter, etc.). A detailed study on the dependence of our matched filter richness estimates on the shape of our filters will be presented in future work.

\subsubsection{The Radial Filter}

$N$-body simulations show that the matter distribution of massive halos can be well described by the so-called Navarro, Frenk, \& White (NFW) profile (see, e.g., Navarro et al. 1995, 1997),

$$
\rho(r) \propto \frac{1}{\left(r / r_{s}\right)\left(1+r / r_{s}\right)^{2}},
$$

where $r_{s}$ is the characteristic scale radius at which the logarithmic slope of the density profile is equal to -2 . The corresponding two-dimensional surface density profile (Bartelmann 1996) is

$$
\Sigma(R) \propto \frac{1}{\left(R / R_{s}\right)^{2}-1} f\left(R / R_{s}\right),
$$

where $R_{s}=r_{s}$ and

$$
f(x)=1-\frac{2}{\sqrt{x^{2}-1}} \tan ^{-1} \sqrt{\frac{x-1}{x+1}} .
$$

This formula assumes $x>1$. For $x<1$, one uses the identity $\tan ^{-1}(i x)=i \tanh (x)$.

Here, we assume that the NFW profile can also reasonably describe the density distribution of galaxies in clusters (Lin \& Mohr 2004; Hansen et al. 2005; Popesso et al. 2007), and follow Koester et al. (2007a) in setting $R_{s}=150 \mathrm{~h}^{-1} \mathrm{kpc}$. In principle, one could optimize the value of this parameter, but we do not expect our final results to be overly sensitive to our chosen value (see, e.g., Dong et al. 2008). Also, in order to avoid the singularity at $R=0$ in the above expression, we set $\Sigma$ to a constant for $R \leqslant R_{\text {core }}=100 h^{-1} \mathrm{kpc}$. This core density is chosen so that the mass distribution $\Sigma(R)$ is continuous. Our results are insensitive to the particular choice of core radius for $R_{\text {core }} \leqslant 200 h^{-1} \mathrm{kpc}$. Finally, the profile $\Sigma(R)$ is truncated at the cluster radius $R_{c}(\lambda)$, and is normalized such that

$$
1=\int_{0}^{R_{c}(\lambda)} d R 2 \pi R \Sigma(R) .
$$

We emphasize that this condition implies that the normalization constant for the density profile is richness dependent, and must be recomputed for each $\lambda$ value when solving for $\lambda$ in Equation (4).

\subsubsection{The Luminosity Filter}

At $z \lesssim 0.3$, the luminosity distribution of satellite cluster galaxies is well represented by a Schechter function (e.g., Hansen et al. 2007) which we write as

$$
\phi(m)=0.4 \ln (10) \phi_{*} 10^{-0.4\left(m-m_{*}\right)(\alpha+1)} \exp \left(-10^{-0.4\left(m-m_{*}\right)}\right) .
$$

We take $\alpha=0.8$ independent of the redshift. The characteristic magnitude, $m_{*}$, is corrected for the distance modulus, $k$-corrected, and passively evolved using stellar population synthesis models described in Koester et al. (2007b). When applying the luminosity filter, $m_{*}$ is chosen from these models, appropriate to the redshift of the cluster under consideration, and the filter is normalized by integrating down to a magnitude corresponding to $0.4 L_{*}$ at the cluster redshift, or an absolute magnitude $M_{i}=-20.25$. The latter is simply a luminosity cut bright enough to make the maxBCG sample volume-limited.

\subsubsection{The Color Filter}

Early-type galaxies are known to dominate the inner regions of low-redshift galaxy clusters (see, e.g., Dressler 1984; Kormendy \& Djorgovski 1989; Hansen et al. 2007). The restframe spectra of these galaxies typically exhibit a significant drop at about $4000 \AA$, that gives early-type galaxies at the same redshift nearly uniformly red colors when observed through filters that encompass this break. In the SDSS survey, the corresponding filters for galaxies at $z \lesssim 0.35$ are $g$ and $r$, and we find that the $g-r$ colors of early-type galaxies are found to be Gaussianly distributed with a small intrinsic dispersion of about 0.05 mag. Consequently, we take the color filter $G(c)$ to be

$$
G(c \mid z)=\frac{1}{\sqrt{2 \pi} \sigma} \exp \left[\frac{(c-\langle c \mid z\rangle)^{2}}{2 \sigma^{2}}\right],
$$

where $c=g-r$ is the color of interest, $\langle c \mid z\rangle$ is the mean of the Gaussian color distribution of early-type galaxies at redshift $z$, and $\sigma$ is the width of the distribution. The mean color $\langle c \mid z\rangle=0.625+3.149 z$ was determined by matching maxBCG cluster members to the SDSS LRG (Eisenstein et al. 2001) and MAIN (Strauss et al. 2002) spectroscopic galaxy samples. The net dispersion $\sigma$ is taken to be the sum in quadrature of the intrinsic color dispersion $\sigma_{\text {int }}$, set to $\sigma_{\text {int }}=0.05$, and the estimated photometric error $\sigma_{m}$. In $g-r$, the typical photometric error on the red-sequence cluster galaxies brighter than $0.4 L_{*}$ is $\sigma_{m} \approx 0.01 \mathrm{mag}$ for $z=0.1$, but can be as large as $\sigma_{m} \approx$ 0.05 mag for $z=0.3$.

\subsubsection{Background Estimation}

To fully specify our filters, we also need to describe our background model. We assume that the background galaxy density is constant in space, so that $b(\mathbf{x})=2 \pi R \bar{\Sigma}_{g}\left(m_{i}, c\right)$ where $\bar{\Sigma}_{g}\left(m_{i}, c\right)$ is the galaxy density as a function of galaxy $i$-band magnitude and $g-r$ color. $\bar{\Sigma}_{g}\left(m_{i}, c\right)$ is estimated by distributing $10^{6}$ random points throughout the same SDSS photometric survey footprint that defines our galaxy sample. All galaxies within an angular separation of $0.05 \mathrm{deg}$ of the random points (about $1 h^{-1} \mathrm{Mpc}$ at $z=0.25$ ) are used to empirically determine the mean galaxy density $\bar{\Sigma}_{g}\left(m_{i}, c\right)$ using a top hat cloud-in-cells (CIC) algorithm (e.g., Hockney \& Eastwood 1981). For our cells, we used 60 evenly spaced bins in $g-r \in[0,2]$ and 40 bins in $i \in[14,20]$. In each two-dimensional bin, the number density of galaxies is normalized by the total number of random points, the width of each color and magnitude bin (0.05 mag and $0.1 \mathrm{mag}$, respectively), and area searched $\left(0.05^{2} \pi\right.$ degrees $)$.

This process creates an estimate of the global background, i.e., the number density of galaxies as a function of color and magnitude in the full SDSS survey. Not surprisingly, a similar result is obtained by binning the whole galaxy catalog in color and magnitude with CIC and dividing by the survey area. However, the procedure we employ above can readily be adapted to returning alternative background estimates, e.g., the local cluster density as a function of redshift, by replacing random points with clusters.

\section{METHODS}

We have now fully specified our richness estimators, except for the values $R_{0}$ and $\beta$ that govern the radius-richness scaling relation. We now discuss how we go about selecting optimal values for these parameters. 
As we mentioned earlier, we wish to find the cluster richness estimator that minimizes the scatter in the richness-mass relation. Cluster mass, however, is not an observable, and thus we must rely on other mass tracers. Here, we use X-ray luminosity $\left(L_{X}\right)$ as our mass proxy, primarily because it is a well known mass tracer (e.g., Reiprich \& Böhringer 2002; Stanek et al. 2006; Rykoff et al. 2008a) that is readily accessible to us and for which we can quickly estimate the scatter for multiple richness measures (see Rykoff et al. 2008b).

We proceed as follows: we begin by defining a coarse grid in $R_{0}$ and $\beta$, given by

$$
\begin{gathered}
R_{0}=\{0.5,0.75,1.0,1.25,1.5\} \\
\beta=\{-0.05,0.05,0.15,0.25,0.35,0.45\},
\end{gathered}
$$

where $R_{0}$ is measured in units of $h^{-1} \mathrm{Mpc}$. Each of these grid points defines a distinct richness estimator through Equation (4). For each grid point, we estimate the corresponding richness for every cluster in the maxBCG catalog. We then select the 2000 richest clusters and calculate the scatter in $L_{X}$ at fixed richness of those top 2000 clusters. Note that, because the rank ordering of the clusters changes as we vary our richness estimate, the clusters used to estimate the scatter in $L_{X}$ varies slightly across the grid. We limit ourselves to the richest 2000 clusters to ensure our results are insensitive to the $N_{200} \geqslant 10$ cut in the maxBCG catalog.

From our measurements of the scatter $\sigma_{\ln L_{X} \mid \lambda}\left(R_{0}, \beta\right)$ at each grid point, we can directly read which parameter combination minimizes the scatter. We emphasize that because the scatter in mass at fixed $L_{X}$ is much lower than the corresponding scatter at fixed richness (E. Rozo et al. 2009, in preparation), for our purposes $L_{X}$ is a nearly perfect mass tracer. We note that the Xray measurements described in Section 2.3 require a minimum aperture of $500 \mathrm{~h}^{-1} \mathrm{kpc}$. For the 2000 richest clusters, this cutoff is only employed when $R_{0}=0.5 h^{-1} \mathrm{Mpc}$ and $\beta \geqslant 0.15$, which is a region of parameter space that already does not appear to have a strong correlation between $L_{X}$ and richness. Therefore, we conclude that the aperture cutoff does not have a significant effect on our results.

To determine the uncertainty in the recovered parameters $R_{0}$ and $\beta$, we need to understand the errors in our measurement of the $L_{X}$-richness scatter. We estimate these errors using bootstrap resampling. We proceed as follows: let $\mu$ be an index that runs over all grid points $\left(R_{0}, \beta\right)$, and let $\sigma_{\mu}$ be the scatter at the $\mu^{\text {th }}$ grid point. We resample (with replacement) the full maxBCG catalog, and measure the scatter $\sigma_{\mu}$ at every grid point. The procedure is iterated 100 times, and the measurements are used to estimate the mean and covariance matrix of $\sigma_{\mu}{ }^{25}$ Assuming that the probability distribution $P\left(\sigma_{\mu}\right)$ is a multivariate Gaussian characterized by the observed mean and covariance matrix, we generate $10^{5}$ Monte Carlo realizations of the scatter, and estimate the fraction of times that each grid point is observed to have the lowest scatter among all grid points.

To use the grid to zero in on a particular value for $R_{0}$ and $\beta$, and to estimate errors in these values, we fit each of the $10^{5}$ realizations of the scatter $\sigma_{\ln L_{X} \mid \lambda}\left(R_{0}, \beta\right)$ with a $2 \mathrm{D}$ parabola. From the fits, we can read off the values of $R_{0}$ and $\beta$ at which

\footnotetext{
25 The measurement of the scatter in $L_{X}$ at fixed richness is very time consuming, and needs to be done independently for every point in the grid. This explains why we restrict ourselves to only 100 bootstrap resamplings.
}

the minimum occurs, giving us $10^{5}$ samplings of the probability distribution of the location of the minimum in parameter space. The probability distribution of the resulting $10^{5}$ minima is exactly what we desired.

As it turns out, and as discussed in Section 5, the coarse grid defined above is too broad for a parabolic fit to adequately describe the function $\sigma_{\ln L_{X} \mid \lambda}\left(R_{0}, \beta\right)$. However, if we restrict ourselves to a smaller region of parameter space near our expected value $\beta=1 / 3$, a quadratic fit becomes adequate. Therefore, we have defined a narrower fine grid,

$$
\begin{gathered}
R_{0}=\{0.9,1.0,1.1,1.2,1.3,1.4,1.5\} \\
\beta=\{0.22,0.26,0.30,0.34,0.38,0.42\}
\end{gathered}
$$

with $R_{0}$ measured in units of $h^{-1} \mathrm{Mpc}$. It is this grid that we use to report our final results and to select the optimal parameters $R_{0}$ and $\beta$.

To summarize, we first do a rough exploration of the parameter space $R_{0}$ and $\beta$ using a coarse grid, and then use a smaller but finer grid to statistically constrain the location of the scatter minimum.

\section{RESULTS}

\subsection{Identification of Low $L_{X}-$ Richness Scatter Regions}

Figure 2 illustrates the probability that each coarse grid point is found to minimize the scatter of the 2000 richest clusters when resampling our data as described in Section 4. For this plot, we have used the full cluster sample, though a similar result holds when using the clean cluster sample. Each square is shaded in gray on a log scale according to the fraction of trials that point is found to have the minimum scatter. The primary feature of this plot is a broad degeneracy region from $\left(R_{0}, \beta\right) \approx(0.8,0.0)$ to $\left(R_{0}, \beta\right) \approx(1.4,0.5)$, corresponding to a scatter $\sigma_{\ln L_{X} \mid \lambda} \approx 0.78$. Note, this scatter is a significant improvement relative to the $L_{X}-$ richness scatter measured for $N_{200}, \sigma_{\ln L_{X} \mid N_{200}}=0.96$. The scatter in $L_{X}$ increases as we move away from the degeneracy region, ranging from $\sigma_{\ln L_{X} \mid \lambda} \sim 0.86$ in the lower right corner of Figure 2 to $\sigma_{\ln L_{X} \mid \lambda}>1.0$ in the upper-left corner. Further discussion of why our new richness estimator results in significantly reduced scatter is presented in Section 6.

Figure 3 shows the probability density of the points in $R_{0}-\beta$ space that minimize the scatter in $L_{X}$ at fixed richness for the fine grid, as estimated through the parabolic fits to the function $\sigma_{\ln L_{X} \mid \lambda}\left(R_{0}, \beta\right)$ described in Section 4 . The solid contours are for the full cluster sample and the dashed contours are for the clean cluster sample. The diagonal degeneracy suggested in the previous plot is now very obvious, especially in the $2 \sigma$ contour. Importantly, both the full and clean samples produce very similar results, although the contours are noticeably smoother for the clean sample. We note that the closing of the $1 \sigma$ contours in the upper right and lower left is likely an artifact of the grid boundaries. As demonstrated in the coarse grid in Figure 2, the degeneracy region extends at least to $\beta \sim 0$ and $\beta \sim 0.5$.

The existence of the degeneracy region is relatively simple to explain. Consider the problem we are trying to address: what is the correct size of a cluster? Roughly speaking, this involves two parts: one, determining the correct cluster size of the average cluster, and two, determining how the cluster size scales with richness as one moves away from the average cluster. The former is much better determined than the latter, so in the 


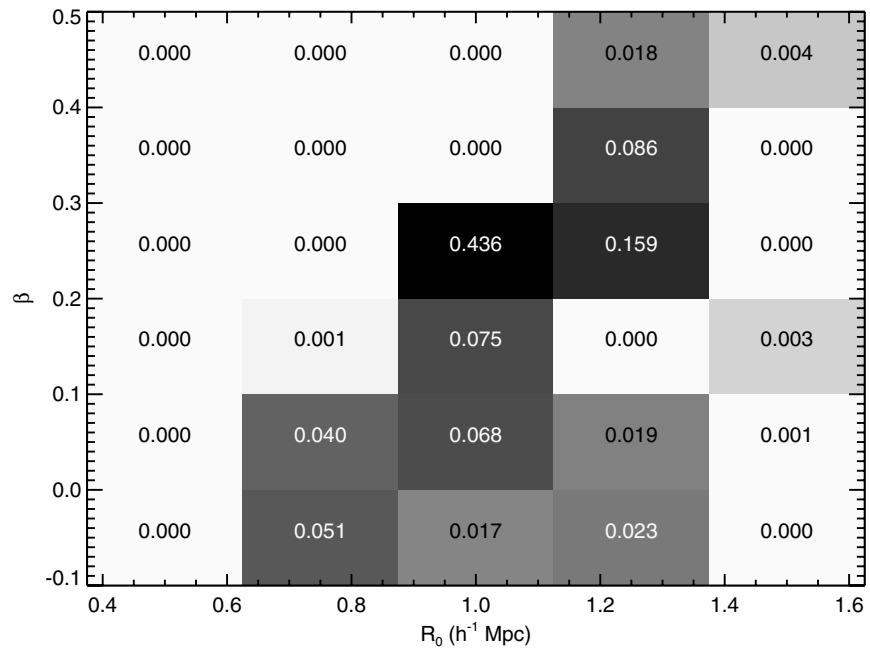

Figure 2. Probability that a given point in the grid minimizes the scatter in $L_{X}$ at fixed richness in the coarse grid. The gray scale varies logarithmically with the probability, which is explicitly quoted in the figure. Note the broad degeneracy region from $\left(R_{0}, \beta\right) \approx(0.8,0.0)$ to $\left(R_{0}, \beta\right) \approx(1.4,0.5)$, where the scatter $\sigma_{\ln L_{X} \mid \lambda} \sim 0.78$.

$\left(R_{0}, \beta\right)$ plane, one typically expects a sharp constraint on the mean cluster radius, and a considerably weaker constraint on the orthogonal direction, corresponding to the scaling of the radius with richness around the statistical pivot point. Thus, we expect the observed degeneracy between $R_{0}$ and $\beta$ to pick out parameter combinations that hold the median cluster radius of the sample fixed.

Figure 3 clearly illustrates that this is the case. In this figure, the diagonal dotted line corresponds to a fixed median cluster radius $\tilde{R}\left(R_{0}, \beta\right)=900 h^{-1} \mathrm{kpc}$, where the function $\tilde{R}\left(R_{0}, \beta\right)$ is defined as the median cluster radius of the 2000 richest clusters. The fact that this contour falls almost exactly along the observed degeneracy between $R_{0}$ and $\beta$ strongly supports our interpretation.

Our argument suggests a way to break the degeneracy between $R_{0}$ and $\beta$. If we can measure the scatter in $L_{X}$ at fixed richness at two very different richness scales, then the mean radius picked out by each of the samples will be substantially different. This, in turn, rotates the degeneracy lines relative to each other, so that the intersection defined by the two samples would cleanly pick out a single value for $R_{0}$ and $\beta$.

We have repeated our analysis on the top 500 and 1000 clusters, but these thresholds are much too close to our reported 2000 clusters to be able to successfully break the observed degeneracy. Ideally, we would repeat our study using the 10,000 or 20,000 richest clusters, thereby guaranteeing a degeneracy region that is significantly rotated relative to that of Figure 3. Unfortunately, performing our scatter analysis on the top 10,000 clusters is not presently possible since the vast majority of this larger cluster sample does not emit sufficiently in X-rays to allow for individual luminosity estimates of the clusters. Furthermore, when choosing more than the top $\sim 3000$ clusters we begin to run into threshold effects due to the initial selection of maxBCG clusters with $N_{200} \geqslant 10$. One might hope instead to repeat our analysis using not the top 10,000 clusters, but rather the top 100 clusters, that is, by limiting ourselves to very rich systems. Unfortunately, this suffers from a different problem: when looking at the top 100 clusters only, the range of richnesses being sampled is much too narrow to allow a simultaneous estimate of the amplitude, slope, and scatter of the $L_{X}$-richness

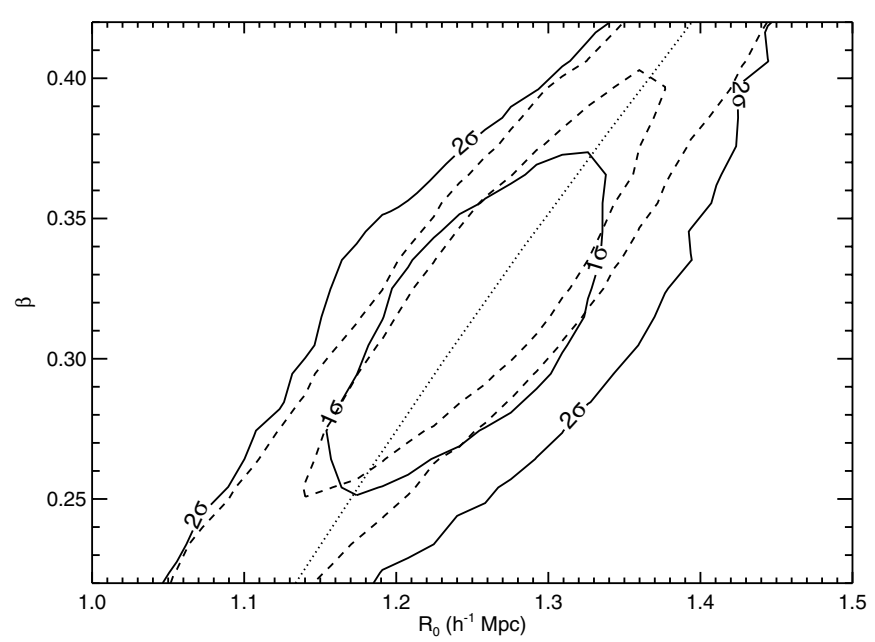

Figure 3. Contour plot of the probability density of the points in $R_{0}-\beta$ space that minimize the scatter $\sigma_{\ln L_{X} \mid \lambda}\left(R_{0}, \beta\right)$. The solid contours show the $1 \sigma$ and $2 \sigma$ contours for the full sample, and the dashed lines show the same contours for the "cleaned" sample (see Section 2.4). The closing of the $1 \sigma$ contours in the upper right and lower left are likely to be an artifact of the grid. The dotted line shows that corresponds to a fixed mean cluster radius $R_{c}=900 h^{-1} \mathrm{kpc}$. All the richness estimators along this line result in the same mean cluster radius, and have therefore very similar richness values.

relation, so performing our analysis using the top 100 clusters only is also not feasible. Thus, for the time being, we must simply accept the existence of a large degeneracy between $R_{0}$ and $\beta$.

\subsection{Selecting an Optimal $R_{0}$ and $\beta$}

Due to the large degeneracy between $R_{0}$ and $\beta$, it is difficult to select any single point in $R_{0}-\beta$ space as optimal. We note, however, that the degeneracy region goes through $\beta=1 / 3$, which is loosely theoretically motivated based on the naive expectation $R^{3} \propto M \propto \lambda$. Since our goal is to define a unique richness measure, we have opted for setting $\beta=1 / 3$. Given that the degeneracy region goes through $\beta=1 / 3$, our choice does not adversely affect the properties of our richness estimator. That is, the scatter for $\beta=1 / 3$ is indistinguishable from that of the best possible value for $\beta$ to within observational uncertainties.

Using a principal component analysis on the best-fit minima that describe the contours in Figure 3, we have calculated the degeneracy axis for each of the full and clean cluster samples. For the full cluster sample we obtain

$$
\ln \left(R_{0} / 1 h^{-1} \mathrm{Mpc}\right)-1.342(\beta-0.33)=0.25 \pm 0.04,
$$

while for the clean cluster sample we find

$$
\ln \left(R_{0} / 1 h^{-1} \mathrm{Mpc}\right)-1.277(\beta-0.33)=0.24 \pm 0.03
$$

We have confirmed that the residuals are Gaussian along most of the degeneracy axis. We quote the degeneracy line in terms of $\ln R_{0}$ and $\beta$ rather than $R_{0}$ and $\beta$ themselves simply because the former results in more accurate extrapolations for $\beta$ values that are very different from $\beta=1 / 3$.

We are encouraged by the fact that the clean and full samples give fully consistent results, thus showing that the known cool core clusters and obvious foreground contamination are not significantly biasing the best combination of $R_{0}$ and $\beta$. Our final choice for $R_{0}$ and $\beta$ is therefore $R_{0}=1.27 h^{-1} \mathrm{Mpc}$ and $\beta=1 / 3$. 
Table 1

Scatter in $L_{X}$ at Fixed Richness, Top 2000 Clusters

\begin{tabular}{lcc}
\hline \hline Richness & Full Sample & Clean Sample \\
\hline$N_{200}$ & $0.95 \pm 0.03$ & $0.86 \pm 0.02$ \\
$N_{200} L_{B C G}^{0.79}$ & $0.84 \pm 0.02$ & $0.78 \pm 0.02$ \\
$\lambda$ & $0.79 \pm 0.02$ & $0.70 \pm 0.02$ \\
$\lambda$ & $0.78 \pm 0.02$ & $0.69 \pm 0.02$ \\
\hline
\end{tabular}

Notes. Except for the last row, $L_{X}$ was measured within a fixed $1 h^{-1} \mathrm{Mpc}$ aperture. The scatter in $L_{X}$ quoted in the last row is different only in that it measured $L_{X}$ within the assigned optical cluster radius $R_{c}(\lambda)$. The combination $N_{200} L_{\text {BCG }}^{0.79}$ was suggested by Reyes et al. (2008) as an improvement over $N_{200}$. The error bars define $68 \%$ confidence intervals.

\subsection{Improvement in the Scatter}

Now that we have a fully specified $R_{0}=1.27 h^{-1} \mathrm{Mpc}$ and $\beta=1 / 3$, and we have measured the matched filter richness of every cluster in the Koester et al. (2007b) sample. Figure 4 shows $L_{X}$ versus $N_{200}$ (top panel) and $L_{X}$ versus $\lambda$ (bottom panel) for the top 3000 richest clusters. Following Rykoff et al. (2008b), the solid points represent detections at the $>1 \sigma$ level, and the empty points represent $1 \sigma$ upper limits. The vertical dotted line represents the cutoff for the top 2000 richest clusters used in this analysis. Though not obviously visible in this plot, the scatter in $\lambda$ is significantly decreased. We note that there are still some significant outliers in the $L_{X}-\lambda$ relation, especially at high $L_{X}$. The red diamonds and blue squares represent clusters that are removed from the clean cluster sample. The red diamonds are clusters whose measured X-ray flux is known to be contaminated by foreground emission from stars, nearby galaxy clusters, or the AGN. The blue squares represent the known cool core clusters. These are, for the most part, significantly brighter than typical maxBCG clusters at similar richness, which is consistent with the hypothesis that the X-ray luminosity of these clusters is boosted by emission from the core.

Table 1 summarizes how the scatter of the 2000 richest clusters varies as we change our richness measure. Here, we consider three richness measures only: $N_{200}$, which is the original richness estimate for maxBCG clusters presented in Koester et al. (2007a); $N_{200} L_{\mathrm{BCG}}^{0.79}$, which was suggested by Reyes et al. (2008) as an improvement over $N_{200}$ by making use of $L_{\mathrm{BCG}}$, the luminosity of the cluster BCG; and our optimized matched filter richness estimator $\lambda$. We see that for both the full and clean samples, our optimized matched filter estimator significantly outperforms both $N_{200}$ and $N_{200} L_{\mathrm{BCG}}^{0.79}$. To quantify the significance of the improvement, we must take into account the fact that the errors are correlated. Following Section 4, we have performed bootstrap resampling on the full catalog and clean catalog, calculating the scatter in the top 2000 clusters for both $\lambda$ and $N_{200}$. For each bootstrap resampling we calculate $r=\sigma_{\ln L_{X} \mid \lambda} / \sigma_{\ln L_{X} \mid N_{200}}$. If the improvement in scatter is not significant, we will find that $r$ is consistent with unity, whereas an improved scatter will result in an $r$ value that is significantly less than $r=1$. We find that $r<1$ at $9 \sigma$ for the full cluster sample, and at $11 \sigma$ for the clean sample.

\section{REDSHIFT DEPENDENCE}

Rykoff et al. (2008b) showed that there is strong redshift evolution in the mean $\left\langle L_{X} \mid N_{200}\right\rangle$ relation of maxBCG clusters. Similar redshift dependence is observed in the velocity dispersion-optical richness relation measured in Becker et al. (2007a). This is best understood as a variation of $N_{200}$ at fixed
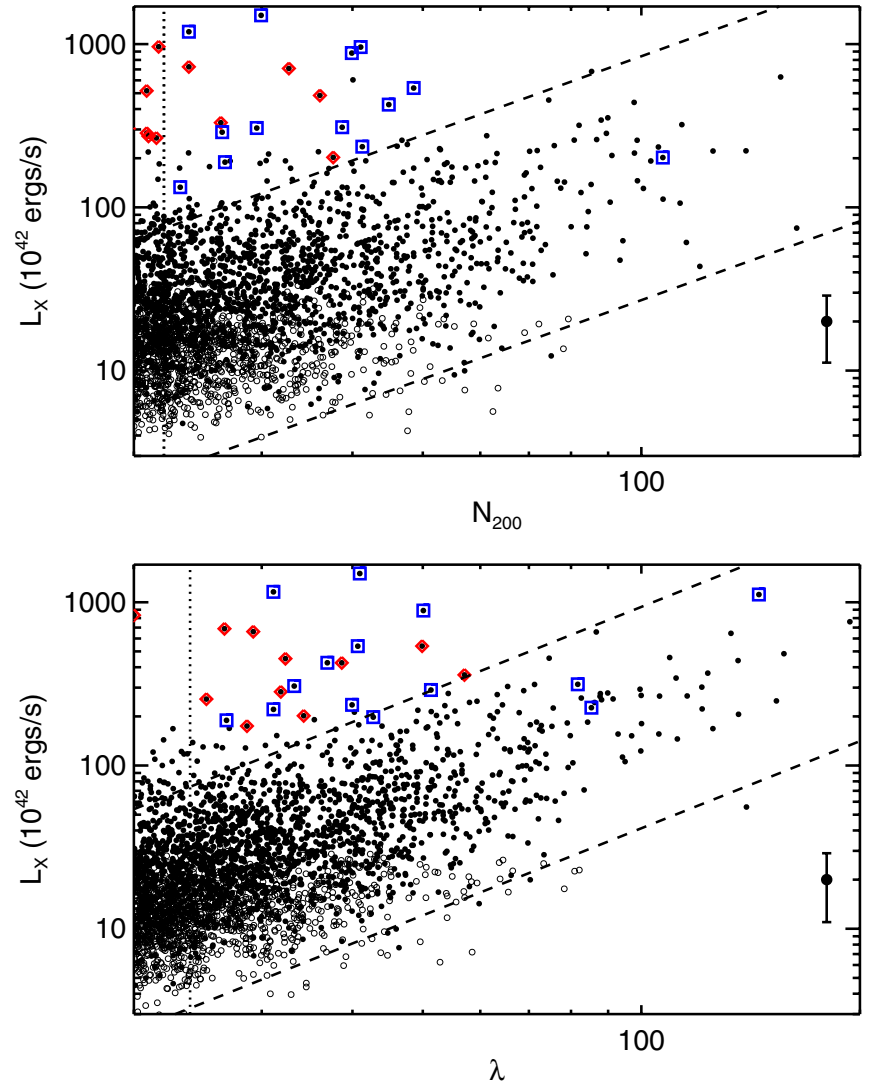

Figure 4. Top panel: $L_{X}$ vs. $N_{200}$ for the 3000 richest clusters. Following Rykoff et al. (2008b), the solid points represent $>1 \sigma$ detections, and the empty circles represent $1 \sigma$ upper limits. The vertical dotted line represents the cutoff for the top 2000 clusters used in the analysis. The dashed lines represent the $\pm 2 \sigma_{\ln L \mid N_{200}}$ scatter constraints. The fictitious data point in the lower right corner shows the typical $L_{X}$ error. Richness errors are very small, typically less than $10 \%$. The red diamonds represent clusters that are excluded from the clean sample because they are obviously contaminated by foreground X-ray emission. The blue squares represent clusters that are excluded from the clean sample because they are known cool core clusters. Bottom panel: $L_{X}$ vs. $\lambda$ for $R_{0}=1.27$, $\beta=1 / 3$ for the 3000 richest clusters; the symbols are the same as for the top panel. Our optimized matched filter richness estimate $\lambda$ is significantly more tightly correlated with $L_{X}$ than $N_{200}$.

(A color version of this figure is available in the online journal.)

mass, with an observed fractional decrease in $N_{200}$ of $30 \%-40 \%$ over the redshift range of the maxBCG catalog. In our previous work, the origin of this redshift dependence was unclear. Here, we demonstrate how the matched-filter richness removes this redshift dependence, and show the pitfalls of a simple richness estimator such as $N_{200}$.

Figure 5 shows the mean $\left\langle L_{X} \mid N_{200}\right\rangle$ relation for maxBCG clusters split into three different redshift bins (solid symbols). Also shown is the mean relation $\left\langle L_{X} \mid \lambda\right\rangle$ for the same three redshift bins (empty symbols). It is obvious from the figure that the redshift evolution in the $L_{X}$-richness relation is significantly weaker for $\lambda$ than it is for $N_{200}$. We have fitted the data with a power-law evolution in redshift, following Rykoff et al. (2008b, Section 5.3):

$$
\left\langle L_{X} \mid N\right\rangle=A\left(\frac{N}{40}\right)^{\alpha}\left(\frac{1+z}{1+\tilde{z}}\right)^{\gamma},
$$

where $\tilde{z}$ is the median redshift of the cluster sample and $N$ is the richness measure of interest. We find that $\gamma=6.0 \pm 0.8$ for $N_{200}$ while $\gamma=0.7 \pm 0.8$ for $\lambda$, consistent with no evolution. 


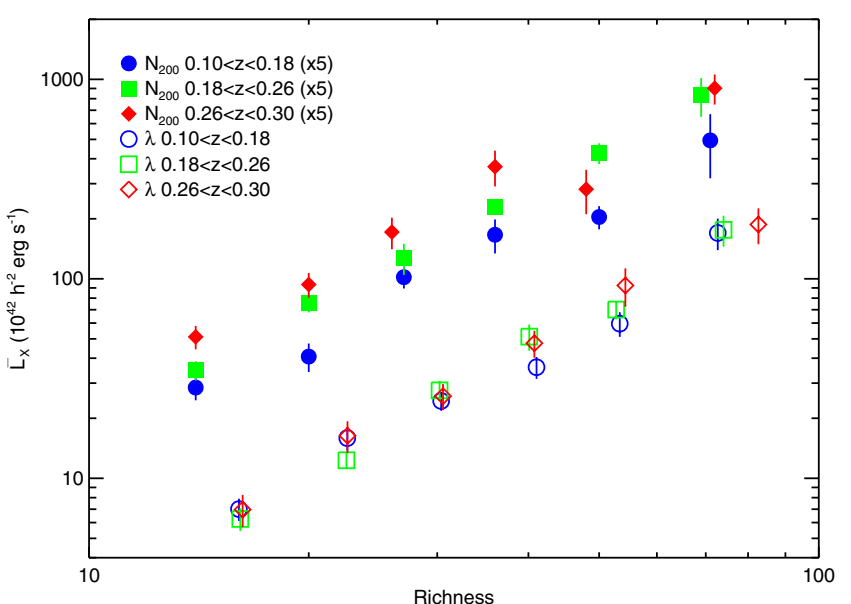

Figure 5. $\left\langle L_{X}\right\rangle$ vs. richness in three different richness bins. The empty points denote the matched filter richness $\lambda$, and the solid points denote the original maxBCG richness $N_{200}$. The three richness bins are $0.10<z<0.18$ (blue circles); $0.18<z<0.26$ (green squares); $0.26<z<0.30$ (red diamonds). The normalization of $\left\langle L_{X}\right\rangle-N_{200}$ has been multiplied by 5 for clarity. It is readily apparent that $N_{200}$ has a strong redshift dependence (Rykoff et al. 2008b; Becker et al. 2007a), while $\lambda$ does not.

(A color version of this figure is available in the online journal.)

Note, however, that even if the relation between $\lambda$ and cluster mass is redshift independent, we expect to see evolution in the $L_{X}-\lambda$ relation due to evolution in the $L_{X}-M$ relation. The expectation for self-similar evolution in $L_{X}$ at fixed mass is that $L_{X} \propto \rho_{c}(z)^{7 / 6}$ for bolometric luminosities, but closer to $\bar{\rho}_{c}^{1.0}$ for soft-band X-ray luminosities (Kaiser 1986). Here, $\rho_{c}$ is the critical density of the universe at redshift $z$. In a $\Lambda$ CDM universe with $\Omega_{m}=0.25, L_{X} \propto \rho_{c}$ is well approximated by $L_{X} \propto(1+z)^{1.10}$, or $\gamma=1.10$, implying our results are also consistent with self-similar evolution.

The striking difference in the evolution in the $L_{X}$-richness relation between $\lambda$ and $N_{200}$ is due to the differences in how $N_{200}$ and $\lambda$ employ galaxy colors when estimating cluster richness. For $\lambda$, a galaxy contributes to the richness by an amount that is proportional to a Gaussian color filter centered on the mean color of red-sequence galaxies at the cluster's redshift. For $N_{200}$, a galaxy contributes to the richness if and only if its color differs from the BCG color by no more than twice the intrinsic width of the ridgeline color width plus the galaxy's photometric error, added in quadrature. That is, $N_{200}$ weighs galaxies according to the probability distribution $p_{\text {top-hat }}(c)$ given by

$$
p_{\text {top-hat }}(c)= \begin{cases}1 & \text { if }\left|c-c_{\mathrm{BCG}}\right| \leqslant \sqrt{\left(2 \sigma_{\text {int }}\right)^{2}+\sigma_{\text {obs }}^{2}} \\ 0 & \text { otherwise }\end{cases}
$$

where $\sigma_{\text {int }}=0.05$ is the intrinsic width of the ridgeline. This is a top-hat distribution in observed color, but the width of the top-hat depends on the photometric error of the galaxy under consideration. Also, note that the center of the color box is not the model $\langle c \mid z\rangle$ quoted earlier, but rather the color of the BCG, which, as we show below, is a very significant difference.

To illustrate how these differences in the color filter result in differences in the evolution and scatter of $\lambda$ and $N_{200}$, we have defined three additional richness measures with key properties bridging those of $\lambda$ and $N_{200}$. Including $\lambda$ and $N_{200}$, the five richness measures considered here are the following.

1. $\lambda$ : the matched filter richness with a variable aperture, as described above, with a Gaussian color filter centered on $\langle c \mid z\rangle$.
2. $\lambda_{B C G}$ : the matched filter richness using the same aperture as with $\lambda$, but with the Gaussian model centered on $c_{\mathrm{BCG}}$.

3. $N_{\text {top-hat,model }}$, a top-hat richness using the $p_{\text {top-hat }}$ formulation above, centered around $\langle c \mid z\rangle$ as in Equation (14), measured on a fixed $1 h^{-1} \mathrm{Mpc}$ scale.

4. $N_{\text {top-hat,BCG }}$, a top-hat richness using the $p_{\text {top-hat }}$ formulation above, centered around $c_{\mathrm{BCG}}$, measured on a fixed $1 h^{-1} \mathrm{Mpc}$ scale. This is similar to the maxBCG $N_{\text {gals }}$ richness, without the additional cut on the $r-i$ color of the member galaxies.

5. $N_{200}$, the original maxBCG richness estimator, measured in a scaled radius $r_{200}^{\text {gals }}$, with the color filter centered on $c_{\mathrm{BCG}}$.

Table 2 shows the scatter (in the top 2000 clusters) and evolution parameters for these various richness estimators. There are two key observations that we can make here. First, when using the top-hat richness, centering around the model color is significantly better than centering on the BCG color, in terms of decreasing both the scatter and evolution of the richness measure. Indeed, our $N_{\text {top-hat,model }}$ richness performs nearly as well as our matched filter richness estimator, both in terms of the scatter and evolution. Second, the smooth Gaussian filter centered on the BCG color works almost as well as the Gaussian filter centered on the model color. This is a significant result, because it implies that not only are the resulting richnesses more robust to moderate changes in the color filter parameters, but also the richness measure itself is also robust to photometric redshift errors. The reason for this robustness is simple: when using a color top-hat selection, using the correct color model is of paramount of importance since miscentering of the top-hat will lead to underestimate of the richness. In the matched filter framework, what is important is the relative galaxy density of the cluster and field components, which can remain high even if the centering of the ridgeline color is slightly displaced. Thus, matched-filter richness estimates are much more robust to small changes in the parameters of the color filter than estimates based on simple color cuts. Because of this robustness, we believe then that even though $N_{\text {top-hat,model }}$ performs similarly to $\lambda, \lambda$ is still a superior richness estimator.

As an illustration of this effect, Figure 6 shows the color distribution of all galaxies brighter than $0.4 L_{*}$ within $1 h^{-1} \mathrm{Mpc}$ (solid black line) of the galaxy cluster SDSS J082026.8+073650.1 at a redshift $z_{\text {spec }}=0.22$. This cluster was selected because of the large discrepancy between $N_{200}$ and $\lambda$. The color of the cluster BCG (solid red line) is significantly redder than the red sequence. The dotted vertical lines show the $\pm\left[\left(2 \sigma_{\text {int }}\right)^{2}+\sigma_{\text {obs }}^{2}\right]^{1 / 2}$ color cut, which does not include the peak of the red sequence. As a result, $N_{200}$ is significantly underestimated in this system. The blue curve shows the same galaxy distribution, but weighing each galaxy by its membership probability as estimated using the matched filter approach. As we can see, the matched filter effectively selects galaxies in the red sequence.

We have demonstrated that the redshift evolution observed in the $L_{X}-N_{200}$ relation is primarily caused by using a top-hat filter centered on the color of the BCG. Why such a choice of color filter results in the strong evolution we observe for $N_{200}$ is a complicated question, with at least two physical mechanisms contributing to the problem at comparable levels. First, there is the fact that even for a correctly centered top-hat filter, a ridgeline galaxy can fall outside the color cuts due to photometric errors. Since photometric errors increase with increasing redshift, a color cut such as that of $N_{200}$ will progressively lose more galaxies as one moves the cluster to higher redshift. Second, the 


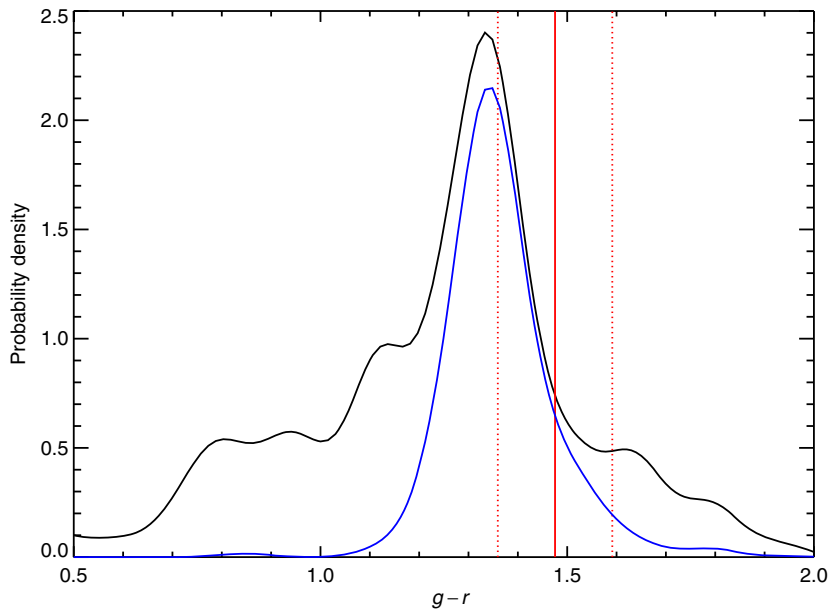

Figure 6. Color distribution of all the galaxies brighter than $0.4 L_{*}$ within $1 h^{-1} \mathrm{Mpc}$ (solid black line) of the central galaxy of cluster SDSS $\mathrm{J} 082026.8+073650.1$ at a redshift of $z_{\mathrm{spec}}=0.22$. The distribution is estimated using a Gaussian Kernel Density Estimator (KDE), with the size of the kernel selected to adequately sample the peak due to ridgeline galaxies. The cluster BCG color (solid red line) is significantly redder than the red sequence (peak of the black distribution). The dotted vertical lines show the $\pm \sqrt{\left(2 \sigma_{\text {int }}\right)^{2}+\sigma_{\text {obs }}^{2}}$ color cut (see text for details) used to estimate $N_{200}$. Note this cut does not include the bulk of the red sequence, implying that the number of red-sequence galaxies is significantly underestimated. The blue curve is the KDE estimate of the galaxy distribution, except every galaxy has been weighted by its membership probability as estimated using the matched filter approach. We can see the matched filter richness estimate selects principally ridgeline galaxies. The slightly lower height of the blue solid curve is primarily due to background subtraction.

(A color version of this figure is available in the online journal.)

Table 2

Scatter $\left(\sigma_{\ln L_{X} \mid N}\right)$ and Redshift Evolution $(\gamma)$

\begin{tabular}{lcc}
\hline \hline \multicolumn{1}{c}{ Richness } & $\sigma_{\ln L_{X} \mid N^{\mathrm{a}}}$ & $\gamma$ \\
\hline$\lambda$ & $0.78 \pm 0.02$ & $0.7 \pm 0.8$ \\
$\lambda_{\text {BCG }}$ & $0.82 \pm 0.02$ & $1.1 \pm 0.8$ \\
$N_{\text {top-hat,model }}$ & $0.80 \pm 0.02$ & $0.5 \pm 0.8$ \\
$N_{\text {top-hat,BCG }}$ & $0.99 \pm 0.02$ & $4.2 \pm 0.7$ \\
$N_{200}$ & $0.95 \pm 0.02$ & $6.0 \pm 0.8$ \\
\hline
\end{tabular}

Note.

${ }^{a}$ For the top 2000 clusters.

$\mathrm{E} / \mathrm{S} 0$ ridgeline is not flat, but has a slight tilt $(\sim-0.04$ mags $\mathrm{mag}^{-1}$ in $g-r$ versus $i$ ), such that brighter galaxies tend to be redder (e.g., Visvanathan \& Sandage 1977; Renzini 2006). By centering the color filter on the BCG-by definition the brightest and usually reddest cluster member-a small richness bias is introduced: clusters with brighter BCGs have a color filter centered redward of the average BCG color. Moreover, recent work by J. Hao et al. (2009, in preparation) shows that with a proper account for photometric errors, the ridgeline tilt evolves with redshift, such that the ridgeline is steeper at $z=0.3$ than at $z=0.1$. Consequently, a BCG-centered color cut becomes increasingly offset from the true mean ridgeline color as we increase redshift. Both of these systematics effects occur with similar magnitude, and act in concert to produce the observed evolution in $N_{200}$. We emphasize, however, that our matched filter richness estimator does not suffer from these systematic effects.

Finally, we can now also explain why $N_{200}$ exhibits stronger

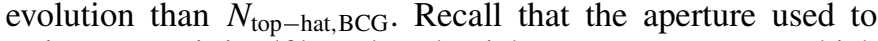
estimate $N_{200}$ is itself based on the richness measure $N_{\text {gals }}$, which is very similar to $N_{\text {top-hat,BCG }}$. Since $N_{\text {top-hat,BCG }}$ systematically underestimates the richness for high redshift clusters due to the increasing tilt of the ridgeline, the aperture $r_{200}^{\text {gals }}$, which scales with $N_{\text {top-hat,BCG }}$, is also underestimated. This compounds the effect of incorrect centering of the color box and results in stronger redshift evolution.

\section{SUMMARY AND CONCLUSIONS}

We have introduced a new matched filter richness estimator $\lambda$ whose correlation with mass as estimated by X-ray luminosities is significantly tighter $\left(\sigma_{\ln L_{X}}=0.69\right.$ versus $\left.\sigma_{\ln L_{X}}=0.86\right)$ than that of $N_{200}$, the original maxBCG richness estimator. Relative to other matched filter estimates, our estimator has two significant differences.

1. The richness is measured on a scale that is optimized in the sense that it minimizes the scatter in $L_{X}$ at fixed richness.

2. In addition to a radial and magnitude filters, we include a color filter. This is of crucial importance for differentiating between member and non-member (projected) galaxies.

The first point is important since we have demonstrated that a poor choice of aperture increases the scatter in mass at fixed richness (see also Popesso et al. 2004; Lopes et al. 2006), while the latter minimizes the impact of projection effects in richness estimates. Of the two, however, the improved treatment of galaxy color is the principal reason for the marked reduction of the scatter in the $L_{X}$-richness relation.

Our procedure for aperture optimization can easily be generalized to any mass tracer for which one can construct a calibrating data set. In our particular case, we minimize the scatter in the $L_{X}-\lambda$ relation by measuring both $L_{X}$ and $\lambda$ within an aperture $R_{c}(\lambda)=R_{0}(\lambda / 100)^{\beta}$, and varying the model parameters $R_{0}$ and $\beta$. Given the small richness range probed by our sample, we have not been able to isolate unique values for $R_{0}$ and $\beta$, finding instead a degeneracy region corresponding to a fixed mean cluster radius for the clusters in the sample. Based on a priori assumptions about the radius-richness scaling, we have fixed $\beta=1 / 3$, which yields a normalization of $R_{0}=1.27 \pm 0.03$. We note, however, that the degeneracy region intersects $\beta=0$ at $R_{0} \approx 850 h^{-1} \mathrm{kpc}$. Although we expect that this fixed scaling will not be ideal at the rich group/poor cluster scale, it does work as a "first guess" richness and may be applicable to future cluster finding techniques. At this point, it is unclear whether the cluster radii selected by our technique reflects a true physical property of the maxBCG clusters, or whether it is driven primarily by a compromise between the increase signal one expects at larger aperture, and the smaller noise one expects for smaller apertures. Regardless of the source, it is likely that similar aperture dependences exist for other mass tracers.

We have also found our new richness estimator has scaling relations whose redshift evolution is much milder than those exhibited by $N_{200}$. This difference arises due to two effects: first, $N_{200}$ uses a top-hat filter to select cluster galaxies, where as our matched filter estimator $\lambda$ uses Gaussian color filters. Second, $N_{200}$ centered its color filter at the color of the BCG, whereas $\lambda$ centered its color using an observationally calibrated color-redshift relation. The fact that the color of the BCG does not always agree with the observationally calibrated redshiftcolor relation leads to a systematic difference between the two richness measures sourced by those galaxies outside the offcenter color cut. Moreover, we also found that the sharp edges of the top-hat filter result in a richness estimator that is very sensitive to the details of the color model, whereas our Gaussian 
filter is much more robust to moderate changes in the model parameters.

Restricting ourselves to the clean cluster sample, which excludes cooling flow clusters and clusters with obvious foreground contamination in their X-ray luminosities, we have found that the scatter in the $L_{X}$-richness relation of the 2000 richest clusters is $\sigma_{\ln L_{X} \mid \lambda}=0.69$ for $\lambda$, compared to $\sigma_{\ln L_{X} \mid N_{200}}=0.86$ for $N_{200}$. Assuming a slope of $\approx 1.6$ for the $L_{X}-M$ relation (Stanek et al. 2006; Rykoff et al. 2008a; Vikhlinin et al. 2009a), these amount to a logarithmic scatter in the mass-richness relation of $\approx 0.43$ and 0.54 , respectively. While this is a very significant improvement, we expect that further tightening of the scatter in mass at fixed richness must be possible. For instance, assuming the intrinsic scatter in the richness-mass relation is Poisson, the logarithmic scatter possible for clusters with 20 galaxies or so should be roughly $\approx 0.2$.

Fortunately, there are still many options left for us to explore in our quest to define optical mass proxies that can be competitive with other mass tracers in terms of the tightness of the correlation with mass. As we have defined it here, our richness estimates only makes use of the number of galaxies in the cluster. One could, for instance, weigh our cluster richness by other optical mass tracers such as the luminosity of the brightest cluster galaxy (Reyes et al. 2008), the abundance of baryons contained in the intracluster light (e.g., Gonzalez et al. 2007), or other aspects of the cluster galaxy morphology (e.g., Bautz-Morgan Type, Bautz \& Morgan 1970). In addition, one could weigh each galaxy's contribution to the richness by physical observables such as galaxy luminosity. Such a luminosity-weighted richness estimate would be a measure of the optical luminosity of the cluster as a whole, and might be better correlated with mass than richness itself (see also Lin et al. 2003; Miller et al. 2005 Popesso et al. 2005). It is also likely that further improvements in richness estimates can arise with more accurate filters, a possibility we intend to explore in future work. Finally, we know that even with today's filters, part of the scatter we observe must be due to systematic effects such as failures of the cluster finding algorithm in identifying the correct center of a cluster, a problem which we have not addressed in this work. For time being, the fact that naive theoretical expectations result in a scatter much lower than previously observed, and the fact that on our first attempt at defining a better richness estimator resulted in a highly significant $(\approx 11 \sigma)$ improvement over $N_{200}$, suggest that the future is rife with opportunities for this kind of work.

E.R. thanks David Weinberg and Christopher Kochanek for interesting discussions and their careful reading of the manuscript. E.S.R. thanks the TABASGO foundation. R.H.W. was supported in part by the U.S. Department of Energy under contract number DE-AC02-76SF00515 and by a Terman Fellowship at Stanford University. T.M. and J.H. gratefully acknowledge support from NSF grant AST 0807304 and DoE Grant DE-FG02-95ER40899. This project was made possible by workshop support from the Michigan Center for Theoretical Physics. A.E. thanks NSF grant AST-0708150.

Funding for the creation and distribution of the SDSS Archive has been provided by the Alfred P. Sloan Foundation, the Participating Institutions, the National Aeronautics and Space Administration, the National Science Foundation, the U.S. Department of Energy, the Japanese Monbukagakusho, and the Max Planck Society. The SDSS Web site is http://www.sdss.org/.

The SDSS is managed by the Astrophysical Research Consortium (ARC) for the Participating Institutions. The Participat- ing Institutions are The University of Chicago, Fermilab, the Institute for Advanced Study, the Japan Participation Group, The Johns Hopkins University, the Korean Scientist Group, Los Alamos National Laboratory, the Max-Planck-Institute for Astronomy (MPIA), the Max-Planck-Institute for Astrophysics (MPA), New Mexico State University, University of Pittsburgh, University of Portsmouth, Princeton University, the United States Naval Observatory, and the University of Washington.

\section{APPENDIX}

\section{A MAXIMUM LIKELIHOOD DERIVATION OF MATCHED FILTER RICHNESS ESTIMATORS}

Here, we derive Equation (2) using a ML approach, focusing first in the case where the filters $u(\mathbf{x} \mid \lambda)$ are richness independent. The derivation is as follows: we pixelize the observable space $\mathbf{x}$ into infinitesimal pixels of "volume" $\Delta \mathbf{x}$ such that every pixel contains at most one galaxy. The likelihood that a given galaxy realization occurs is simply

$$
\mathcal{L} \propto \prod_{\text {occupied }}(\lambda u+b) \Delta \mathbf{x} \prod_{\text {empty }}(1-(\lambda u+b) \Delta \mathbf{x}),
$$

where the first product is over all occupied pixels, while the second product is over all empty pixels. We have neglected terms that do not depend on $\lambda$ as they will not contribute to the ML richness estimator. Setting $\partial \ln \mathcal{L} / \partial \lambda=0$, and taking the limit $\Delta \mathbf{x} \rightarrow 0$ we find that the ML richness estimator $\hat{\lambda}_{\mathrm{ML}}$ is given by the solution to

$$
1=\sum \frac{u}{\lambda u+b},
$$

where the sum is over all galaxies in the cluster field. This expression is identical to our naive richness estimator from Equation (2).

We wish to briefly consider how richness-dependent filters $u(\mathbf{x} \mid \lambda)$ affect the ML richness estimator. To do this, we go back to Equation (A1). Taking the derivative of the log-likelihood with respect to $\lambda$ and setting it to zero we find that the generalization of Equation (2) is given by

$$
1+\int d \mathbf{x} \lambda \frac{\partial u}{\partial \lambda}=\sum \frac{u+\lambda(\partial u / \partial \lambda)}{\lambda u+b} .
$$

We emphasize that the integral over $\mathbf{x}$ and the derivative $\partial / \partial \lambda$ do not always commute. Indeed, consider the approach taken in this paper, in which $u$ is taken to have a finite spatial extent of radius $R_{c}$, which is itself linked to richness via Equation (3). The fact that $u$ is zero for $R>R_{c}(\lambda)$ implies that the integration region for $u$ is $\lambda$ dependent, and thus the integral and derivative signs do not commute.

To assess the impact of a richness-dependent profile, we consider here a simple isothermal filter $u(R \mid \lambda)=1 / R_{c}$, where $R_{c}(\lambda)$ is given by Equation (3). ${ }^{26}$ For this filter, we have then

$$
\lambda \frac{\partial u}{\partial \lambda}=\lambda \frac{\partial u}{\partial R_{c}} \frac{\partial R_{c}}{\partial \lambda}=-\beta u,
$$

where $\beta$ is the slope of the radius-richness relation in Equation (3). Our expression for the ML richness estimator becomes

$$
(1-\beta)=\sum_{R<R_{c}(\lambda)} \frac{(1-\beta) u}{\lambda u+b} .
$$

\footnotetext{
26 The two-dimensional density profile is, of course, $\Sigma(R) \propto 1 / R$, but the
} radial probability density is $u(R)=2 \pi R \Sigma(R)=1 / R_{c}$. 
We see that the $1-\beta$ prefactors cancel on both sides of the equation, and thus our final expression for the ML richness estimator for $\lambda$ is still given by Equation (4), even though $u$ is explicitly richness dependent. This suggests that our naive estimator is in general very close to the true ML estimator. We defer a detailed study of whether the more complicated structure of the true ML richness estimator for more elaborate cluster profiles can lead to a significant improvement over the naive richness estimator from Equation (4) to future work.

\section{REFERENCES}

Abell, G. O. 1958, ApJS, 3, 211

Abell, G. O., Corwin, H. G., Jr., \& Olowin, R. P. 1989, ApJS, 70, 1

Adelman-McCarthy, J. K., et al. 2006, ApJS, 162, 38

Adelman-McCarthy, J. K., et al. 2008, ApJS, 175, 297

Bahcall, N. A. 1981, ApJ, 247, 787

Bartelmann, M. 1996, A\&A, 313, 697

Bauer, F. E., Fabian, A. C., Sanders, J. S., Allen, S. W., \& Johnstone, R. M. 2005, MNRAS, 359, 1481

Bautz, L. P., \& Morgan, W. W. 1970, ApJ, 162, L149

Becker, M. R., et al. 2007a, ApJ, 669, 905

Becker, M. R., et al. 2007b, ApJ, 669, 905

Böhringer, H., Burwitz, V., Zhang, Y.-Y., Schuecker, P., \& Nowak, N. 2005, ApJ, 633,148

Böhringer, H., et al. 2004, A\&A, 425, 367

Bond, J. R., Cole, S., Efstathiou, G., \& Kaiser, N. 1991, ApJ, 379, 440

Bower, R. G., Lucey, J. R., \& Ellis, R. S. 1992, MNRAS, 254, 601

Bramel, D. A., Nichol, R. C., \& Pope, A. C. 2000, ApJ, 533, 601

Chen, Y., Reiprich, T. H., Böhringer, H., Ikebe, Y., \& Zhang, Y.-Y. 2007, A\&A, 466, 805

Cuesta, A. J., Prada, F., Klypin, A., \& Moles, M. 2008, MNRAS, 389, 385

Dai, X., Kochanek, C. S., \& Morgan, N. D. 2007, ApJ, 658, 917

Dong, F., Pierpaoli, E., Gunn, J. E., \& Wechsler, R. H. 2008, ApJ, 676, 868

Dressler, A. 1984, ARA\&A, 22, 185

Edge, A. C., Stewart, G. C., Fabian, A. C., \& Arnaud, K. A. 1990, MNRAS, 245,559

Eisenstein, D. J., et al. 2001, AJ, 122, 2267

Fukugita, M., Ichikawa, T., Gunn, J. E., Doi, M., Shimasaku, K., \& Schneider, D. P. 1996, AJ, 111, 1748

Gilbank, D. G., Bower, R. G., Castander, F. J., \& Ziegler, B. L. 2004, MNRAS, 348,551

Gladders, M. D., \& Yee, H. K. C. 2000, AJ, 120, 2148

Gladders, M. D., \& Yee, H. K. C. 2005, ApJS, 157, 1

Gladders, M. D., Yee, H. K. C., Majumdar, S., Barrientos, L. F., Hoekstra, H., Hall, P. B., \& Infante, L. 2007, ApJ, 655, 128

Gonzalez, A. H., Zaritsky, D., \& Zabludoff, A. I. 2007, ApJ, 666, 147

Goto, T., et al. 2002, AJ, 123, 1807

Gunn, J. E., et al. 2006, AJ, 131, 2332

Haiman, Z., Mohr, J. J., \& Holder, G. P. 2001, ApJ, 553, 545

Hansen, S. M., McKay, T. A., Wechsler, R. H., Annis, J., Sheldon, E. S., \& Kimball, A. 2005, ApJ, 633, 122

Hansen, S. M., Sheldon, E. S., Wechsler, R. H., \& Koester, B. P. 2007, arXiv:0710.3780

Henry, J. P., Evrard, A. E., Hoekstra, H., Babul, A., \& Mahdavi, A. 2009, ApJ, 691,1307

Hockney, R. W., \& Eastwood, J. W. 1981, Computer Simulation Using Particles (New York: McGraw-Hill)

Hogg, D. W., Finkbeiner, D. P., Schlegel, D. J., \& Gunn, J. E. 2001, AJ, 122 , 2129

Holder, G., Haiman, Z., \& Mohr, J. J. 2001, ApJ, 560, L111

Hu, W., \& Cohn, J. D. 2006, Phys. Rev. D, 73, 067301

Jenkins, A., Frenk, C. S., White, S. D. M., Colberg, J. M., Cole, S., Evrard, A. E., Couchman, H. M. P., \& Yoshida, N. 2001, MNRAS, 321, 372

Johnston, D. E., et al. 2007, arXiv:0709.1159

Kaiser, N. 1986, MNRAS, 222, 323

Kelly, B. C. 2007, ApJ, 665, 1489

Kepner, J., Fan, X., Bahcall, N., Gunn, J., Lupton, R., \& Xu, G. 1999, ApJ, 517, 78

Kochanek, C. S., White, M., Huchra, J., Macri, L., Jarrett, T. H., Schneider, S. E., \& Mader, J. 2003, ApJ, 585, 161
Koester, B. P., et al. 2007a, ApJ, 660, 239

Koester, B. P., et al. 2007b, ApJ, 660, 221

Kormendy, J., \& Djorgovski, S. 1989, ARA\&A, 27, 235

Kravtsov, A. V., Vikhlinin, A., \& Nagai, D. 2006, ApJ, 650, 128

Lima, M., \& Hu, W. 2004, Phys. Rev. D, 70, 043504

Lima, M., \& Hu, W. 2005, Phys. Rev. D, 72, 043006

Lin, Y.-T., \& Mohr, J. J. 2004, ApJ, 617, 879

Lin, Y.-T., Mohr, J. J., \& Stanford, S. A. 2003, ApJ, 591, 749

Lopes, P. A. A., de Carvalho, R. R., Capelato, H. V., Gal, R. R., Djorgovski, S. G., Brunner, R. J., Odewahn, S. C., \& Mahabal, A. A. 2006, ApJ, 648, 209

Lupton, R., Gunn, J. E., Ivezić, Z., Knapp, G. R., \& Kent, S. 2001, in ASP Conf. Ser. 238, Astronomical Data Analysis Software and Systems X, ed. F. R. Harnden, Jr., F. A. Primini, \& H. E. Payne (San Francisco, CA: ASP), 269

Majumdar, S., \& Mohr, J. J. 2004, ApJ, 613, 41

Mantz, A., Allen, S. W., Ebeling, H., \& Rapetti, D. 2008, MNRAS, 387, 1179

Martínez, H. J., \& Muriel, H. 2006, MNRAS, 370, 1003

Martínez, H. J., Zandivarez, A., Domínguez, M., Merchán, M. E., \& Lambas, D. G. 2002, MNRAS, 333, L31

Maughan, B. J. 2007, ApJ, 668, 772

Miller, C. J., et al. 2005, AJ, 130, 968

Muzzin, A., Yee, H. K. C., Hall, P. B., \& Lin, H. 2007, ApJ, 663, 150

Navarro, J. F., Frenk, C. S., \& White, S. D. M. 1995, MNRAS, 275, 56

Navarro, J. F., Frenk, C. S., \& White, S. D. M. 1997, ApJ, 490, 493 (NFW)

O'Hara, T. B., Mohr, J. J., Bialek, J. J., \& Evrard, A. E. 2006, ApJ, 639, 64

Peres, C. B., Fabian, A. C., Edge, A. C., Allen, S. W., Johnstone, R. M., \& White, D. A. 1998, MNRAS, 298, 416

Pier, J. R., Munn, J. A., Hindsley, R. B., Hennessy, G. S., Kent, S. M., Lupton, R. H., \& Ivezić, Ž. 2003, AJ, 125, 1559

Popesso, P., Biviano, A., Böhringer, H., \& Romaniello, M. 2007, A\&A, 464, 451

Popesso, P., Biviano, A., Böhringer, H., Romaniello, M., \& Voges, W. 2005, A\&A, 433, 431

Popesso, P., Böhringer, H., Brinkmann, J., Voges, W., \& York, D. G. 2004, A\&A, 423, 449

Postman, M., Lubin, L. M., Gunn, J. E., Oke, J. B., Hoessel, J. G., Schneider, D. P., \& Christensen, J. A. 1996, AJ, 111, 615

Press, W. H., \& Schechter, P. 1974, ApJ, 187, 425

Reiprich, T. H., \& Böhringer, H. 2002, ApJ, 567, 716

Renzini, A. 2006, ARA\&A, 44, 141

Reyes, R., Mandelbaum, R., Hirata, C. M., Bahcall, N., \& Seljak, U. 2008, MNRAS, 390, 1157

Rozo, E., Dodelson, S., \& Frieman, J. A. 2004, Phys. Rev. D, 70, 083008

Rozo, E., et al. 2008, arXiv:0809.2797

Rozo, E., et al. 2007, arXiv:astro-ph/0703571

Rozo, E., et al. 2009, arXiv:0902.3702

Rykoff, E. S., et al. 2008a, MNRAS, 387, L28

Rykoff, E. S., et al. 2008b, ApJ, 675, 1106

Sheldon, E. S., et al. 2007a, arXiv:0709.1153

Sheldon, E. S., et al. 2007b, arXiv:0709.1162

Sheth, R. K., \& Tormen, G. 2002, MNRAS, 329, 61

Smith, J. A., et al. 2002, AJ, 123, 2121

Stanek, R., Evrard, A. E., Böhringer, H., Schuecker, P., \& Nord, B. 2006, ApJ, 648, 956

Strauss, M. A., et al. 2002, AJ, 124, 1810

Tinker, J. L., Kravtsov, A. V., Klypin, A., Abazajian, K., Warren, M. S., Yepes, G., Gottlöber, S., \& Holz, D. E. 2008, ApJ, 688, 709

Tucker, D. L., et al. 2006, Astron. Nach., 327, 821

Vikhlinin, A., et al. 2009a, ApJ, 692, 1033

Vikhlinin, A., et al. 2009b, ApJ, 692, 1060

Visvanathan, N., \& Sandage, A. 1977, ApJ, 216, 214

Voges, W., et al. 1999, A\&A, 349, 389

Warren, M. S., Abazajian, K., Holz, D. E., \& Teodoro, L. 2006, ApJ, 646, 881

Weinmann, S. M., van den Bosch, F. C., Yang, X., \& Mo, H. J. 2006, MNRAS, 366,2

Wu, H.-Y., Rozo, E., \& Wechsler, R. H. 2008, ApJ, 688, 729

Yee, H. K. C., \& Ellingson, E. 2003, ApJ, 585, 215

Yee, H. K. C., \& López-Cruz, O. 1999, AJ, 117, 1985

York, D. G., Adelman, J., Anderson, J. E., Anderson, S. F., \& Annis, J. the SDSS collaboration. 2000a, AJ, 120, 1579

York, D. G., et al. 2000b, AJ, 120, 1579 